\title{
Diffuse Cluster-Like Radio Emission in Poor Environments
}

\author{
Shea Brown \& Lawrence Rudnick \\ Department of Astronomy, University of Minnesota, Minneapolis, MN 55455
}

\begin{abstract}
We present a study of the spectral, polarimetric, morphological and environmental properties of the diffuse radio source $0809+39$ using observations taken with the Westerbork Synthesis Radio Telescope, the Very Large Array, and archival optical and X-ray data. The source has two distinct diffuse, steep-spectrum components, one in the north that is highly polarized, and a linear southern component undetected in polarization. We discuss several plausible origins for each component, and conclude that the northern bright polarized component is most likely a radio relic associated with a poor $\mathrm{z} \sim 0.2$ cluster of galaxies, with a radio/X-ray luminosity ratio two orders of magnitude above typical values. The southern component is aligned with a more extended filament of galaxies $\sim 5 \mathrm{Mpc}$ long at $\mathrm{z} \sim 0.04$. Deep optical and X-ray follow-ups are still needed in order to confirm and understand the physical origins of the synchrotron emission. Whatever the details of these origins, $0809+39$ highlights the utility of synchrotron radiation for illuminating the diffuse components of low density environments unrelated to rich clusters.
\end{abstract}

Subject headings: galaxies: clusters: general-large scale structure of universe-radiation mechanisms: non-thermal-radio continuum: general-techniques: polarimetric

\section{Introduction}

In recent years, large-scale diffuse radio sources have been discovered to be associated with the intracluster medium (ICM) of over 50 clusters of galaxies. These features are unique probes of the non-thermal particle populations and magnetic fields within the cluster, and are believed to be important tracers of merger/formation dynamics. The majority of these sources were found by searching the environs of rich galaxy clusters for diffuse radio emission unassociated with active AGN (Giovannini et al. 1999; Kempner et al. 2004). But is there also a magnetized, relativistic plasma associated with lower density regions of the cosmic web, such as groups and filaments of galaxies? Cosmological simulations predict complicated networks of accretion/merger shocks in these low density regions, which could accelerate particles, compress magnetic fields, and illuminate magnetized plasma if it exists (e.g., Miniati et al. 2001; Rvu et al. 2003; Pfrommer. Enßlin \& Springel 2008). Unbiased searches for synchrotron signatures of these shocks are needed to confirm these predictions. 
One such diffuse source, of ambiguous origin, is 0809+39 (Delain \& Rudnick 2006), discovered through a blind search for diffuse radio emission in the WENSS survey (Rudnick. Delain \& Lemmerman 2006). The system showed no obvious associations with an active radio galaxy. Several poor clusters were found in the vicinity, which suggests a similar origin to radio relic and halo sources. However, lacking any evidence for cluster X-ray emission, its radio luminosity far exceeded the standard radio/x-ray ratios for these classes of sources (e.g., Giovannini \& Feretti 2004; Rudnick \& Lemmerman 2008).

We present a systematic study of the spectral, polarimetric, morphological and environmental properties of 0809+39 using observations taken with the Westerbork Synthesis Radio Telescope (WSRT) and the Very Large Array (VLA). We explore the origin of the diffuse radio emission and evaluate where $0809+39$ falls in the parameter space of known extragalactic radio sources. We discuss observations and data reduction in $\S 2$, and in $\S 3$ we outline image production and analysis, including our application of Rotation Measure Synthesis (Brentjens \& de Bruyn 2005). In §4 we present archival optical and x-ray data, and in $\S 5$ we discuss the implications of our findings, followed by a summary of our key messages.

For calculations in this paper, we assume $H_{o}=70, \Omega_{\Lambda}=0.7, \Omega_{M}=0.3$. We define the optically thin synchrotron spectrum as $F_{\nu} \propto \nu^{\alpha}$ throughout.

\section{Observations \& Data Reduction}

\subsection{Observations}

The diffuse source 0809+39 was observed for 13 hours over two nights in P-band (350 MHz) with the Westerbork Synthesis Radio Telescope (WSRT) in January of 2006. The array was in the maxi-short configuration with the shortest baseline being $36 \mathrm{~m}$. One primary flux and one polarized calibrator (as a pair) were observed at the beginning and end of each night. For the primary flux calibrators we observed 3C147 and 3C295, and for the polarized calibrators we observed DA240 and 3C303. We used the WSRT wide band correlator to cover a frequency range from 310-390 MHz with eight $10 \mathrm{MHz}$ wide bands, each with 64 channels and full Stokes parameters. We applied a Hanning taper to the spectral data, and every other channel was selected for analysis yielding an effective spectral resolution of about $0.31 \mathrm{MHz}$. After removing the end channels in each band and editing for strong RFI, 197 channels remained in the final analysis, for a total bandwidth of $61 \mathrm{MHz}$.

The VLA observations were taken for 2.8 hours in December of 2005 in the D configuration, and the flux calibrators $3 \mathrm{C} 48$ and $3 \mathrm{C} 286$ were used. The data were taken using the spectrometer with no cross-had polarizations. We analyzed only the pseudo-continuum data for this analysis, with a frequency of $1.4649 \mathrm{GHz}$, a bandwidth of $12.5 \mathrm{MHz}$, and no polarization information. 


\subsection{Total Intensity Calibration}

The calibration and reduction of the WSRT and VLA data was performed using the NRAO's Astronomical Image Processing System (AIPS). For the WSRT data, the total intensity in each of the 8 bands was calibrated independently using standard procedures and the fluxes in the VLA calibrator manual for 3C147 and 3C295. The VLA pseudo-continuum data set was calibrated using standard procedures. We did several iterations of amplitude and phase self-calibration on each data set.

\subsection{Polarization Calibration}

Due to the fact that WSRT observes with orthogonal linear feeds (X and Y), the polarization calibration in classic AIPS involved several non-standard steps. WSRT polarization leakage terms are highly frequency dependent, so after the total intensity calibration, each channel was split into an independent uv-data set and the leakage terms were then solved for using the AIPS task LPCAL. Stokes $\mathrm{Q}$ values were calculated from $(\mathrm{YY}-\mathrm{XX}) / 2$, while the Stokes $\mathrm{U}$ values are $-(\mathrm{XY}+\mathrm{YX}) / 2$. An additional correction is needed for Stokes $\mathrm{U}$, to remove the instrumental phase offset between the $\mathrm{X}$ and $\mathrm{Y}$ receivers using the polarized calibrators. Faraday rotation causes the Stokes $\mathrm{Q}$ and U amplitudes of the polarized calibrators to oscillate across the band. The X-Y phase offset was found for each channel by forcing the vector averaged Stokes $U=U\left(\lambda^{2}\right)$ visibilities for the polarized calibrators to match (offset by a quarter wavelength) the $Q\left(\lambda^{2}\right)$ sine wave formed by Faraday rotation. The observed sign of the Faraday rotation for 3C303 $\left(+13 \mathrm{rad} \mathrm{m}^{-2}\right.$, Kronberg et al. 1977) forced Stokes U values to be a quarter wavelength after the Stokes Q values with increasing wavelength, breaking the final sign ambiguity and finishing the polarization calibration. However,

we note that the intrinsic position angle of the polarization is still highly uncertain due to errors in the rotation measure, as discussed in $§ 3.3$.

\section{Image Production \& Analysis}

\subsection{Initial Spectral Cube}

After calibration, we created I, Q, and U images for each of the 197 WSRT channels. All images and self-calibrations were done in AIPS with the tasks IMAGR and CALIB respectively. Each channel image was cleaned with IMAGR with 70,000 clean components and a gain of 0.1. The synthesized beam varied from $89^{\prime \prime} \times 52^{\prime \prime}$ to $108^{\prime \prime} \times 60^{\prime \prime}$ across the band, so all of the images were uv-tapered and restored to a common resolution of $108^{\prime \prime} \times 60^{\prime \prime}$. Typical rms noise levels for the single-channel $\{\mathrm{I}, \mathrm{Q}, \mathrm{U}\}$ maps were $\{1.0,0.5,0.5\} \mathrm{mJy} /$ beam, respectively. 


\subsection{Total Intensity}

\subsubsection{WSRT I-Map}

The final WSRT I map is shown in Figure1, This map is the simple average of all the individual channel I maps $\left(108^{\prime \prime} \times 60^{\prime \prime}\right.$ beam). The average frequency is $351 \mathrm{MHz}$ and the observed noise is $\sigma \sim 188 \mu \mathrm{Jy} /$ beam. The diffuse emission is seen to have two distinct components which we have labeled $\mathrm{N}_{\text {Diff }}$ and $\mathrm{S}_{\text {Diff }}$. At this resolution, $\mathrm{N}_{\text {Diff }}$ partially blends into the compact sources $S_{1}$ and $S_{2}$. As shown in $\S 3.2 .2$, some of these "compact" sources also have substructure. Flux and size properties for both diffuse sources are summarized in Table 1 . We describe all the discrete radio sources in the next subsection.

\subsubsection{VLA I-Map}

Figure 2 shows our VLA I map, which has a noise level of $90 \mu \mathrm{Jy} /$ beam and a $40^{\prime \prime} \times 40^{\prime \prime}$ restoring beam. We have labeled the discrete radio sources, and have shown VLA FIRST (Becker et al. 1995) images of some of the more interesting ones. The sources F1-F7 are adjacent to or embedded in the diffuse emission. F2 is a 66 mJy wide-angle tailed (WAT: Owen \& Rudnick 1977) radio galaxy at $\mathrm{z}=0.196$ (from SDSS 1 York et al. 2000; Stoughton et al. 2002), and F3 and F5 are identified with SDSS galaxies with photometric redshifts of $\mathrm{z}=0.249$ and $\mathrm{z}=0.255$, respectively. In $\mathrm{S}_{D i f f}$, the source F6 is marginally detected in FIRST (not shown), and is coincident with a $\mathrm{z}=0.04$ galaxy in SDSS. F7 is a FIRST radio point source (not shown) with no optical identification in SDSS. The apparent bridge of emission between $\mathrm{N}_{\text {Diff }}$ and $S_{1}$ peaks on a spiral galaxy at $\mathrm{z}=0.041$ (see Figure 9) and is most likely diffuse disk emission from that galaxy.

\subsubsection{Spectral Index}

For the spectral analysis we matched the uv-range of the VLA and WSRT data before comparison. The spectral index $(\alpha)$ map (Figure 3) was created in AIPS using the task COMB. Any pixel that was not at least $10 \sigma$ above the noise in either map was blanked. Both sources are steep spectrum, and Table 1 lists the integrated $\alpha$ obtained from fitting the total flux of each component at $1.4 \mathrm{GHz}$ and $351 \mathrm{MHz}$ to a power law.

\footnotetext{
${ }^{1}$ http://www.sdss.org/
} 


\subsection{Polarization}

\subsubsection{Rotation Measure Synthesis}

With the 197 channels and $\sim 61 \mathrm{MHz}$ total bandwidth of our WSRT P-band data, it is possible to simultaneously determine the rotation measure distribution within each beam and remove the effects of bandwidth depolarization. When searching for polarized synchrotron emission, which is often at very low surface brightness levels, one would like to observe over a large bandwidth $(\Delta \nu)$ to maximize the signal/noise ratio. However, this normally results in depolarization from the vectoral cancellation of the Stokes Q and U signals that have been Faraday rotated from one side of the band to the other. Some radio telescopes allow for a large observing bandwidth to be split into many narrow channels (Westerbork being one of them), so an obvious solution would be to make an image of the polarized amplitude in each channel and average them together. Unfortunately, polarized galactic foreground emission is ubiquitous (e.g., Reich 2006). This is actually above the surface brightness of some diffuse extragalactic regions of interest, so adding the scalar polarization intensity of each channel will cause the galactic emission to add coherently as well. This increases the background and drowns out the desired diffuse extragalactic source.

Brentjens \& de Bruyn (2005) presented a new technique to eliminate the confounding effects of intervening Faraday rotation (bandwidth depolarization and galactic foreground contamination) by searching "rotation measure space" for polarized emission. The technique proceeds as follows: assume a rotation measure for the source, then derotate the polarization vector in each individual channel (to a reference wavelength $\lambda_{o}$ ) to correct for this and make a polarization map from the average of the derotated channels. If the source in fact had the assumed rotation measure, the channels would add coherently, allowing for the full sensitivity of the entire bandwidth. The resulting map at a given Faraday depth, $\phi$, is approximately given by

$$
F(\phi)=\frac{1}{N} \sum_{j=1}^{N} P_{j} e^{-2 i \phi\left(\lambda_{j}^{2}-\lambda_{o}^{2}\right)}
$$

where $\mathrm{N}$ is the number of maps (channels) used and $P_{j}=Q_{j}+i U_{j}$ is the complex polarization at channel $\mathrm{j}$. The units of $F(\phi)$ are $\mathrm{Jy} /$ beam/rmtf, where rmtf stand for the Rotation Measure Transfer Function. The RMTF is the response of a source with emission at a single Faraday depth. If one were able to sample an infinite range of $\lambda^{2}$, the RMTF would be a delta function in RM space, but incomplete sampling induces side-lobe structures in a manner similar to side-lobes in the beam pattern of an interferometer due to incomplete sampling of the uv-plane.

The key step is to apply Eq. (1) for a wide range of rotation measures, creating a rotation measure cube (RM-Cube), and search for coherent structures within this cube. We processed the $197 \mathrm{Q}$ and U maps in IDL utilizing Eq. (1) to create the final RM-Cube, with Faraday depths running from $-200 \mathrm{rad} \mathrm{m}^{-2}$ to $+200 \mathrm{rad} \mathrm{m}^{-2}$ in steps of $1 \mathrm{rad} \mathrm{m}^{-2}$. This method has been successfully used to detect very diffuse polarized emission in the region of the Perseus cluster 
(de Bruyn \& Brentjens 2005).

Near values of $\phi=0$, polarized emission from our own galaxy fills the field of view. This emission has a typical surface brightness of $\sim 1 \mathrm{mJy} / \mathrm{beam} / \mathrm{rtmf}$, and in the vicinity of $0809+39$ it peaks at $\phi_{\text {Gal }} \sim+6 \mathrm{rad} \mathrm{m}^{-2}$. The polarized emission in $\mathrm{N}_{\text {Diff }}$ peaks near $+12 \mathrm{rad} \mathrm{m}^{-2}$, though there is still a significant amount of galactic emission present at this Faraday depth. At values of $|\phi|>30 \mathrm{rad} \mathrm{m}^{-2}$, very little of the galactic emission remains. As a result, the rms noise of the images decreases with increasing $|\phi|$, to $\sim 30 \mu \mathrm{Jy} /$ beam/rmtf for $|\phi|>100 \mathrm{rad} \mathrm{m}^{-2}$.

Some of the emission that de Bruyn \& Brentiens (2005) detected in the field of the Perseus cluster with RM-Synthesis was later found to likely be Galactic in origin (Brentjens 2007). In the case of $0809+39, \mathrm{~N}_{\text {Diff }}$, while not segregated significantly from the Galactic emission in Faraday depth, is an order of magnitude stronger in surface brightness, making a Galactic origin unlikely.

We can also use this RM-Cube to find Faraday spectra, which is just $F(\phi)$ from Equation 1 for a single pixel or region in the sky. Figure 4 shows the average spectrum of a $1.8^{\prime} \times 1.8^{\prime}$ region centered on $\mathrm{N}_{\text {Diff }}$, along with the RMTF for our frequency sampling. The Faraday spectrum of $\mathrm{N}_{\text {Diff }}$ is very close to a "point source" in Faraday space, especially when compared to a typical Galactic spectrum also shown in Figure 4. The diffuse galactic radiation along the line of sight to 0809+39 has emission at multiple Faraday depths. This galactic signal must also be present in our spectrum of $\mathrm{N}_{\text {Diff }}$, but is lower than the side-lobe level of $\mathrm{N}_{\text {Diff }}$ itself.

\subsubsection{Position Angle}

We used the NRAO VLA Sky Survey (NVSS: Condon et al. 1998) polarization image of $0809+39$, along with our measured RM, to find the absolute position angle $\chi$ of $\mathrm{N}_{\text {Diff }}$. Figure 5 shows NVSS total intensity contours and magnetic field orientation (which is $\chi \pm 90^{\circ}$ ), corrected for the average $\phi$ of $\mathrm{N}_{\text {Diff }}$. Due to the difference in resolution between the RM-Cube and the NVSS, and the fact that the gradient in $\phi$ across the source translates into only a $\delta \chi \sim 7^{\circ}$, we did not do a pixel-by-pixel de-rotation. The magnetic field runs along the long axis of $\mathrm{N}_{D i f f}$, and shows evidence of following the curvature in the southern end.

Our Faraday corrected WSRT magnetic field vectors are plotted in Figure 6, We obtained the polarization from the complex RM-Cube at $\phi=+12 \mathrm{rad} \mathrm{m}^{-2}$, where $\mathrm{N}_{\text {Diff }}$ peaks. Given the uncertainties in absolute position angle when derotating from $\lambda=90 \mathrm{~cm}$ to $\lambda=0$ (e.g., $\sigma_{\chi} \approx 140^{\circ}$ when $\sigma_{\phi}=3 \mathrm{rad} \mathrm{m}^{-2}$ ), we globally rotated the vectors so that the magnetic field orientation of $\mathrm{N}_{\text {Diff }}$ matches that seen in Figure 5. We placed the polarized intensity cut-off such that some of the diffuse Galactic emission can be seen. One can see the discontinuity in angle between the galactic polarization and the brighter $\mathrm{N}_{\text {Diff }}$ emission. 


\subsubsection{Fractional Polarization}

The detection of $\mathrm{N}_{\text {Diff }}$ in the NVSS survey allows for examination of the degree of depolarization. The fractional polarization, $\mathrm{m}$, decreases from $m \sim 45-55 \%$ at $21 \mathrm{~cm}$ (matched to the WSRT resolution) to $m \sim 20 \%$ at $92 \mathrm{~cm}$. Assuming for a moment that this depolarization is due only to internal Faraday de-polarization (Burn 1966; Cioffi \& Jones 1980), we calculate the necessary internal Faraday rotation to be $\phi_{i n} \approx 2 \mathrm{rad} \mathrm{m}^{-2}$. The upper limit intrinsic width of $\mathrm{N}_{\text {Diff }}$ is $\approx 7-9 \mathrm{rad} \mathrm{m}^{-2}$, so the depolarization could be due to internal Faraday de-polarization. At $351 \mathrm{MHz}, \mathrm{S}_{\text {Diff }}$ is not polarized at a $3 \sigma$ level of $9 \%$.

\section{X-ray \& Optical Identification}

We now turn our attention to the optical and X-ray environment of $0809+39$. Since all radio halo and relic sources found thus far exist in or adjacent to the hot gas of galaxy clusters, we searched for thermal emission from X-ray clusters in the vicinity of $0809+39$ and investigated the surrounding optical field.

\subsection{X-Ray}

Figure 7 shows the ROSAT broad (0.1-2.4 keV) continuum emission in the region of $0809+39$ with VLA L-band radio contours overlayed. To put the low observed brightness of the X-ray field of 0809+39 in context, we have also plotted three X-ray selected clusters at three different redshifts. The clusters are $\{$ RXCJ2324.3+1439, RXCJ1353.0+0509, RXCJ2155.6+1231\} with redshifts of $\{0.042,0.079,0.192\}$ and X-ray luminosities of $\mathrm{L}_{X}=\{0.97,1.97,5.51\} \times 10^{44} h_{70}^{-2} \mathrm{erg} \mathrm{s}^{-1}$, respectively (Popesso et al. 2004). The cluster redshifts were selected to mimic the relevant systems we have identified in $\$ 4.2$. It is clear that no diffuse X-ray emission like that present in the X-ray selected clusters is present in the ROSAT $0809+39$ field.

Figure 8 shows VLA contours over XMM EPIC grayscale as well as XMM contours over SDSS R grayscale from an XMM observation of the nearby galaxy UGC 4229 (P.I. Matteo Guainazzi) where $0809+39$ was toward the edge of the field. There is clear emission from the WAT, but there are also several peaks in the region of $\mathrm{N}_{\text {Diff }}$. Peak 2 is located on the WAT. Peak 1 is coincident with an SDSS photometric object at $z=0.313 \pm 0.1$, and peak 4 is coincident with an SDSS photometric object at $z=0.272 \pm 0.06$. Peak 3 is offset $\sim 10^{\prime \prime}$ from a 2MASS galaxy at $z=0.02$, which is also the FIRST source F1 in Figure 2,

After subtracting out the four point sources, we found no evidence of excess diffuse emission. Using webPIMMS2 we calculate a $3 \sigma$ upper limit X-ray luminosity of $\mathrm{L}_{X}(0.1-2.4 \mathrm{keV})=1 \times 10^{43} \mathrm{erg} \mathrm{s}^{-1}$

\footnotetext{
${ }^{2}$ http://heasarc.nasa.gov/Tools/w3pimms.html
} 
at an assumed redshift of $\mathrm{z}=0.2$.

\subsection{Optical}

As one would expect from a region that spans more than $400^{\prime \prime}$ on the sky, the optical field of $0809+39$ contains multiple, overlapping redshift systems. This is illustrated by Figure 9, which shows an SDSS mosaic R image with VLA contours. To set the scale, the large spiral galaxies in this image are at a redshift of roughly $\mathrm{z}=0.04$. We begin by outlining the known and cataloged optical systems in this region, then focus our attention on the systems we believe are most likely associated with $\mathrm{N}_{\text {Diff }}$ and $\mathrm{S}_{\text {Diff }}$.

Delain \& Rudnick (2006) suggested an association of both $\mathrm{N}_{\text {Diff }}$ and $\mathrm{S}_{\text {Diff }}$ with a grouping of galaxies at $\mathrm{z} \sim 0.04$. A $z=0.04063$ group (16 members) is reported by Miller et al. (2005) from the SDSS cluster catalog C4, and Merchán \& Zandivarez (2005) find a group at z=0.040346 (19 members) from DR3. Gal et al. (2003) also detected $\mathrm{z} \sim 0.073$ and $\mathrm{z} \sim 0.11$ clusters in this region. These are plotted in Figure 10. From Figures 7 and 8 one can see that none of these have associated $\mathrm{X}$-ray emission.

Due to the presence of the WAT at $\mathrm{z}=0.2$, we consider this redshift system as likely associated with $\mathrm{N}_{\text {Diff }}$ (see arguments in $§ 5.1$ ). To assess the significance of the clustering at this redshift, we used the SDSS photometric galaxy database (Adelman-McCarthy et al. 2007) and made a histogram in redshift of all the galaxies within a radius of $6^{\prime}(\sim 1 \mathrm{Mpc}$ at $\mathrm{z}=0.2)$ from the WAT. We then subtracted a histogram (normalized to the same number of total counts) of a roughly $2 \times 2$ degree field around the WAT, in order to subtract out any systematics in SDSS's photometric redshifts. The results are displayed in Figure 11, Figure 12 shows the spatial distribution of galaxies (number of galaxies/pixel at the redshift of the WAT $\mathrm{z}=0.2 \pm 0.05$ ) from the SDSS photometric data. A weak clustering of galaxies is seen around the WAT. We also show in Figures 11 and 12 , for comparison, the results from two X-ray selected clusters at similar redshifts (discussed in §5.1). To assess the relative richness of this group/cluster, we followed the analysis used in Gal et al. (2003) for estimating the richness of clusters found in the POSS-II survey. We took the number of galaxies (above a background value determined from the surrounding $2 \times 2$ degree field) within $\sim 1 \mathrm{Mpc}$ with an absolute $\mathrm{R}$ magnitude of $-22.53<\mathrm{M}_{R}<-19.53$ taken from the SDSS photometric data. The grouping of galaxies associated with the WAT has a richness of 16 , which is on the poorer end of the overall distribution of richnesses in DPOSS (Gal et al. 2003). We should note that the cluster at $\mathrm{z}=0.073$ detected by Gal et al. (2003) is also very close to $\mathrm{N}_{\text {Diff }}$, and is just as likely to be associated with the diffuse emission as the WAT grouping of galaxies, but the issues discussed in $\S 5$ apply to either case. The cluster at $\mathrm{z}=0.073$ had a richness of 24.9 , also significantly poor, and roughly corresponding to an Abell richness class of $\mathrm{R}<0$ (Abell et al. 1989; Gal et al. 2003).

If we allow for the proximity of $\mathrm{S}_{\text {Diff }}$ with $\mathrm{N}_{\text {Diff }}$ to be coincidental, then identifying the optical system that is physically connected with $\mathrm{S}_{D i f f}$ is not straightforward. The three likely possibilities 
are the $\mathrm{z} \sim 0.04,0.073$, and 0.2 groupings of galaxies. We visually searched the spatial distribution of galaxies from the SDSS photometric catalog to find a redshift where there was significant clumping in or near $\mathrm{S}_{\text {Diff }}$, without success. The spectroscopic database of galaxies in SDSS, while much sparser, does reveal a $\sim 5 \mathrm{Mpc}$ filament of galaxies surrounding $\mathrm{S}_{\text {Diff }}$ at redshifts of $0.0352<\mathrm{z}$ $<0.0441$ (Figure 13). Many of these galaxies are those that make up the two groups previously detected in this region (Miller et al. 2005; Merchán \& Zandivarez 2005), but looking at a wider field reveals the larger filament. We performed the same richness analysis on the filament (centered on $\mathrm{RA}=122.224, \mathrm{DEC}=38.883,1 \mathrm{Mpc}$ radius) as we did with the WAT group, and found a richness of 13. Figure 14 shows a wedge diagram of redshift vs. RA for SDSS galaxies with spectra in a roughly $4 \times 4$ degree field around $0809+39$. We have indicated the range of galaxy redshifts that are plotted in Figure 13. Groupings of galaxies at higher redshift were not correlated spatially with $\mathrm{S}_{\text {Diff }}$.

\section{Physical Origin of the Radio Emission}

We now turn our attention to the physical origin of the diffuse emission. Though we cannot strictly rule out a Galactic origin for either source, the emission is not morphologically similar and is far brighter than typical diffuse galactic emission in this region. We hereafter assume that both components are of extragalactic origin. There are many different types of extragalactic large-scale diffuse radio emission (e.g., Kempner et al. 2004), but they in general fall within one of two basic classes: 1) Those directly powered by current AGN activity; 2) Those associated with processes in the intracluster medium (ICM). We include in the second class emission related to processes in the intergalactic plasma of galaxy filaments (Kim et al. 1989; Giovannini et al. 1990; Bagchi et al. 2002; Kronberg et al. 2007). In the vast majority of cases, determining the identification is straightforward. Either the diffuse region is directly connected (via jets or filamentary bridges) to an active radio galaxy, or there is a rich cluster of galaxies nearby whose potential well (highlighted by $\sim 10^{8} \mathrm{~K}$, X-ray emitting gas) provides an obvious energy source for particle acceleration (via gravitational collapse/accretion $->$ shocks/turbulence etc).

Both sources in 0809+39 are unique in that neither of these conditions apply (see Delain \& Rudnick 2006 for two similar sources). There are no cataloged X-ray emitting clusters nearby, only groups and poor clusters of galaxies. An AGN origin for these sources is also not obvious. In this paper we narrow our analysis to the two most likely (or least improbable) sources for the radio emission.

\subsection{Northern Component}

In this section, we: 1) Rule out $\mathrm{N}_{\text {Diff }}$ being an extended radio lobe directly powered by the WAT; 2) Give evidence that $\mathrm{N}_{\text {Diff }}$ is a classical "radio relic"; 3) Compare $\mathrm{N}_{\text {Diff }}$ with other relics and conclude that it has an anomalously high radio/X-ray luminosity ratio; 4) Determine that the 
X-ray luminosity is appropriate for this optical richness, and is thus not to blame for the abnormal luminosity ratio; 5) Examine possible sources of the relativistic electrons such as direct acceleration from the thermal plasma, adiabatic compression (only) of fossil WAT plasma, and reacceleration of fossil WAT plasma. We conclude that the most likely source is reacceleration of fossil plasma from past WAT activity.

We first consider whether $\mathrm{N}_{\text {Diff }}$ could be lobe emission from an AGN. The nearby WAT (F2) is the only reasonable candidate as an AGN source. However, they are spatially well separated, with a peak to peak distance $>500 \mathrm{kpc}$ at $\mathrm{z}=0.2$. While the FWHM of the long axis of $\mathrm{N}_{\text {Diff }}$ is $200^{\prime \prime}$, there are faint wings that extend out to $600^{\prime \prime}$. This corresponds to $\sim 2 \mathrm{Mpc}$ at $\mathrm{z}=0.2$, which is a size comparable to that of a giant radio galaxy (GRG). However, its morphology is unlike any other GRG known (e.g., Machalski et al. 2001). We can also consider the lifetime of particles emitting at $1.4 \mathrm{GHz}$. The minimum energy magnetic field for $\mathrm{N}_{\text {Diff }}$ is $\mathrm{B}_{\text {min }} \sim 0.6 \mu \mathrm{G}$, which fixes the Lorentz factor at $\gamma \sim 2 \times 10^{4}$ and results in inverse Compton losses dominating the lifetime. From Sarazin (1999)

$$
t_{I C}=2.3 \times 10^{12} \gamma^{-1}(1+z)^{-4} y r
$$

which for $\mathrm{N}_{\text {Diff }}$ is $t_{I C} \sim 10^{7.7} \mathrm{yr}$. Relaxing the minimum energy requirement for magnetic field strength, we can calculate the maximum lifetime for any electron radiating at $1.4 \mathrm{GHz}$ following the prescription of Sarazin (1999). Assuming a redshift of $\mathrm{z}=0.2$ yields a maximum lifetime of $\mathrm{t}_{\max } \sim 10^{8} \mathrm{yr}$, similar to that calculated above using $\mathrm{B}_{\min }$.

If we assume a $1 \mathrm{keV}$ gas temperature and only hydrogen gas the sound crossing time is $\mathrm{t}_{s c} \sim 10^{9.1} \mathrm{yr}$. Therefore the timescale for $1.4 \mathrm{GHz}$ IC losses will be much shorter than the diffusion/sound-crossing time (from the WAT to $\mathrm{N}_{\text {Diff }}$ ). If the WAT was the original source for the $\mathrm{N}_{\text {Diff }}$ plasma, e.g. from an earlier outburst, then there must have been some re-acceleration or adiabatic enhancement. This is no surprise because this has been a longstanding result for radio halos and relics.

There are several lines of evidence that point toward $\mathrm{N}_{\text {Diff }}$ being a radio "relic" source associated with a poor cluster at $\mathrm{z} \sim 0.2: 1$ ) Both the clumping in redshift and the existence of the WAT (Owen \& Rudnick 1977) indicate the presence of a cluster; 2) WATs are also known to be associated with merger dynamics (Pinknev 1993; Gomez et al. 1997; Roettiger et al. 1996; Loken et al. 1995; Pinkney et al. 2000; Blanton et al. 2003). Additional supporting evidence for merger activity near $\mathrm{N}_{\text {Diff }}$ comes from the multiple X-ray peaks seen in Figure 8 , one of which is coincident with a $\mathrm{z}=0.27$ SDSS galaxy. Currently all known radio relic or halo sources have been found in/near clusters in a disturbed dynamical state (Feretti 2006); 3) The long axis of the diffuse emission is perpendicular to the line connecting $\mathrm{N}_{\text {Diff }}$ and the WAT, typical of relic sources; 4) The diffuse emission is highly polarized, also typical of radio relic sources (e.g. Giovannini \& Feretti 2004); 5) The magnetic fields are parallel to the long axis of the emission, suggesting shock compression. 
All of these point toward $\mathrm{N}_{\text {Diff }}$ being either a Radio Phoenix or Radio Gischt (Kempner et al. 2004), depending on whether the "seed" plasma came from an extinct radio galaxy lobe or was initially accelerated at a cluster accretion shock, respectively. The curvature of the WAT (from Figure 2) is toward the North, suggesting infall from the South. With a longest linear extent of $\sim 2$ $\mathrm{Mpc}, \mathrm{N}_{\text {Diff }}$ is comparable to larger relic sources around rich clusters of galaxies (Giovannini et al. 1999). For radio relics where the spectral index distribution is known, the edge farthest from the cluster is always sharper and has a flatter spectrum (Giovannini \& Feretti 2004). Though $\mathrm{N}_{\text {Diff }}$ does not exhibit this behavior (see Figure 3), we should note that the source is only a little more than a single WSRT beam thick in the transverse direction. Therefore a higher resolution spectral index map is needed to confirm this.

We can now ask how $\mathrm{N}_{\text {Diff }}$ compares to other observed radio relics. Radio halos, diffuse radio emission centered on some clusters of galaxies, are known to exhibit a good correlation between their radio luminosity and the X-ray luminosity of their associated cluster (e.g., Feretti 2003, 2006). A similar but weaker correlation for radio relics has been claimed by Giovannini \& Feretti (2004), who quoted $P_{1.4 G H z} \propto 10^{K} L_{x}$, where $\mathrm{K}$ ranged from 0.8 to 2.2. We have compiled a relatively complete list of known relics with available $1.4 \mathrm{GHz}$ flux measurements and plotted their radio luminosities vs. X-ray luminosities in Figure 15 (compiled from Giovannini et al. 1991, 1999; Kempner \& Sarazin 2001; Govoni et al. 2001; Slee et al. 2001; Govoni et al. 2005). $\mathrm{N}_{\text {Diff }}$ is at least an order of magnitude too luminous (under-luminous) in the radio (X-ray). We examine several possible explanations as to why the radio or X-ray emission from the relic source $\mathrm{N}_{\text {Diff }}$ is not what we would expect from the radio/X-ray luminosity relation for rich galaxy clusters. From the observed correlation, $\mathrm{N}_{\text {Diff }}$ should have an $L_{x} \sim 10^{44-45} \mathrm{erg} \mathrm{s}^{-1}$ from 0.1-2.4 keV, but from XMM observations we measure a $3 \sigma$ upper limit of $L_{x} \approx 1 \times 10^{43} \mathrm{erg} \mathrm{s}^{-1}$. We examine the two quantities in this relation separately, starting with the $\mathrm{X}$-ray luminosity.

It is possible that the grouping of galaxies that $\mathrm{N}_{\text {Diff }}$ is associated with is massive enough to emit the expected amount of X-rays (i.e. $\sim 10^{44-45} \mathrm{erg} \mathrm{s}^{-1}$ at $0.1-2.4 \mathrm{keV}$ ), but for some reason they are not observed. Perhaps the grouping will have the "correct" X-ray luminosity eventually, but we have caught it in a very early evolutionary state where the thermal gas has not reached the needed density or temperature to emit sufficiently in the X-rays. The optical properties of the WAT group $(\$ 4.2)$, however, are not representative of massive X-ray emitting clusters (see, e.g., Ledlow et al. 2003 for optical vs. X-ray properties of Abell clusters). To further show this, we examined the optical properties of the X-ray selected 3 clusters RXCJ1327.0+0211 and RXCJ2155.6+1231, both of which have a similar redshift $\{0.259,0.192\}$ to the WAT and a $\mathrm{L}_{x}$ $=\{1.67,1.12\} \times 10^{45} \mathrm{erg} \mathrm{s}^{-1}$, respectively (Popesso et al. 2004). For the optical data we again used the SDSS photometric galaxy database and performed the same analysis as with the WAT system (§4.2). Figure 11 shows the histograms and Figure 12 the distribution of galaxies with the same contour levels as the WAT system. The X-ray clusters show much stronger clustering than the

\footnotetext{
${ }^{3}$ We used X-ray selected clusters to avoid pre-selecting optically rich clusters.
} 
WAT system, both spatially and in redshift. Using the same richness analysis that we used for the WAT group, RXCJ2155.6+1231 and RXCJ1327.0+0211 have richnesses of 42 and 53, respectively, consistent with large X-ray luminous clusters (e.g. Ledlow et al. 2003). The lack of X-ray emission in the WAT group, therefore, is consistent with its poor optical properties, and cannot explain the discrepant radio/X-ray luminosity ratio seen in Figure 15.

We now examine the radio luminosity, which is apparently two orders of magnitude too luminous given the observed correlation for radio relics. We proceed under the assumption that $\mathrm{N}_{\text {Diff }}$ is related to the presence of a shock, as we argued earlier, and attempt to understand the source of the relativistic electrons that are causing the synchrotron emission. The two likely possibilities are that the electrons were accelerated directly out of the thermal plasma by diffusive shock acceleration (DSA), or the seed electrons came from fossil plasma from past AGN activity and were re-accelerated and/or adiabatically enhanced by the shock.

Miniati et al. (2001) performed a cosmological simulation that included only DSA of cosmic rays from the thermal environment and did not include fossil AGN plasma. They report a correlation between $1.4 \mathrm{GHz}$ radio luminosity from primary electrons (those accelerated at shocks) and cluster temperature (see Figure 6 of that paper) that is roughly consistent with the observed correlation for radio relics shown in Figure 15. The radio/X-ray luminosity ratio of $\mathrm{N}_{\text {Diff }}$ is therefore inconsistent with the results of Miniati et al. (2001). We can examine the conditions under which their simulations would have produce the observed $\mathrm{P}_{\nu} / \mathrm{L}_{X}$ ratio of $\mathrm{N}_{\text {Diff }}$. Using observed $\mathrm{L}_{X}$ vs. $\mathrm{T}_{\text {cluster }}$ relations (Hartley et al. 2008) and theoretical expectations for low-density environments (Ryu et al. 2003), we assume a $\mathrm{T}_{\text {cluster }}<1 \mathrm{keV}$. Miniati et al. (2001) would then predict $\mathrm{P}_{\nu}(1.4 \mathrm{GHz}) \approx 1 \times 10^{22} \mathrm{~W} \mathrm{~Hz}^{-1}$, assuming an acceleration efficiency of $10^{-4}$ and an electron to proton injection ratio of $\mathrm{R}_{e / p}=0.01$. Therefore, either the acceleration efficiency needs to be $\sim 0.01$ or $\mathrm{R}_{e / p} \sim 1$ in order for Miniati et al. (2001) to reproduce the $\mathrm{P}_{\nu} / \mathrm{L}_{X}$ ratio of $\mathrm{N}_{\text {Diff. }}$. It is thus physically possible to use DSA out of the thermal gas to create $\mathrm{N}_{\text {Diff }}$, but it is not clear why the efficiency or $\mathrm{R}_{e / p}$ should be so anomalous in this region.

The other possible source of seed electrons is fossil or "relic" plasma from past AGN activity, presumably from the nearby WAT. There are two possible mechanisms for reviving old plasma. The shock can either adiabatically enhance the relativistic particles and magnetic fields of the relic plasma only (e.g., Enßlin \& Gopal-Krishna 2001), or the shock can reaccelerate the particles as well.

In the case of pure adiabatic compression, let us compare the energy content of the current WAT radio lobes and $\mathrm{N}_{\text {Diff }}$. From the FIRST data, the eastern lobe of the WAT has a minimum energy magnetic field of $\mathrm{B}_{\min } \approx 2.7 \mu \mathrm{G}$ and a total energy of $\mathrm{E} \approx 6 \times 10^{57} \mathrm{erg}$. $\mathrm{N}_{\text {Diff }}$ has $\mathrm{B}_{\min } \approx$ $0.6 \mu \mathrm{G}$ and $\mathrm{E} \approx 3.4 \times 10^{59} \mathrm{erg}$. It appears that the energy contained in $\mathrm{N}_{\text {Diff }}$ cannot be explained by adiabatically compressing an extinct radio lobe similar to the current lobes of the WAT. This does not rule out this scenario however, since the current activity of the WAT may not be indicative of past activity, and WATs in general are known to have energies of the same order as $\mathrm{N}_{\text {Diff }}$ (e.g., 


\section{De Young 1984).}

The reacceleration of the fossil electrons can further increase the emissivity of the relic plasma (Blandford \& Eichler 1987; Micono et al. 1999; Markevitch et al. 2005), potentially by an order of magnitude, depending on the pre-shock spectral index and the shock compression ratio R. From the spectral index of $\mathrm{N}_{\text {Diff }}, \alpha=-1.12$, we can find the shock compression ratio $\mathrm{R}=\frac{\alpha-1}{\alpha+1 / 2}=3.4$ (Bell 1978; Drurv 1983) and Mach number $\mathrm{M} \approx 2.1$. The Mach number is a reasonable one for this environment (e.g., Ryu et al. 2003). In short, energetically it is not out of the question that the radio emission $\mathrm{N}_{\text {Diff }}$ was created by shock compressed fossil plasma from the WAT, assuming that the WAT's past activity was stronger than it is currently.

Given that DSA of electrons from the thermal plasma reproduces the observed radio vs. Xray luminosity correlation for radio relics, from which $\mathrm{N}_{\text {Diff }}$ is a clear outlier, we conclude that reacceleration and/or adiabatic compression of fossil plasma from the WAT source is a more likely origin for the radio emission in $\mathrm{N}_{\text {Diff }}$. Due to the fact that current activity of the WAT is not energetically comparable to $\mathrm{N}_{\text {Diff }}$, we must invoke more powerful activity in the past in order to make the adiabatic compression/reacceleration hypothesis work. Had we associated $\mathrm{N}_{\text {Diff }}$ with the $\mathrm{z} \sim 0.07$ group of galaxies ( $\$ 4.2$, Figure 10), the $\mathrm{P}_{\nu} / \mathrm{L}_{X}$ ratio would still have been two orders of magnitude too large for the observed correlation for radio relics. Our arguments would have proceeded the same way, except we would have needed to invoke past activity from an undetected AGN to explain the discrepant radio luminosity.

\subsection{Southern Component}

Many of the same issues that we found with $\mathrm{N}_{\text {Diff }}$ arise when we consider possible origins for

$\mathrm{S}_{\text {Diff }}$. If we consider an AGN origin, the morphology of $\mathrm{S}_{\text {Diff }}$ (Figure 2) is reminiscent of a WAT source centered on the FIRST radio source F7. F7, however, does not have an optical counterpart in SDSS. If it is an unidentified WAT, its redshift is likely to be z $>0.5$ (Schmidt et al. 2006), making its total linear extent $>3 \mathrm{Mpc}$. Figure 16 shows a plot of $1.4 \mathrm{GHz}$ radio power vs. linear extent for a sample of WAT sources from Pinkney et al. (2000). If $\mathrm{S}_{\text {Diff }}$ were indeed at a redshift of $\mathrm{z}>0.5$, it would have a linear size that far exceeds typical WATs in this sample. It is also possible that one of the $\mathrm{z}=0.04$ galaxies that makes up the filament (Figure 13) hosts an AGN that created the extended emission, but none of the galaxies at this redshift (with spectra in SDSS) show signs of AGN activity. However, the AGN could have been disrupted and disappeared leaving the lobe emission behind (e.g., Parma et al. 2007), as long as this happens on a timescale less than the 1.4 $\mathrm{GHz}$ electron maximum lifetime of $10^{8} \mathrm{yr}$ (see discussion in §5.1). Assuming $\mathrm{S}_{\text {Diff }}$ is at a redshift of $\mathrm{z}=0.04$, its total energy would be $\mathrm{E} \approx 7.9 \times 10^{57} \mathrm{erg}$ and the minimum energy magnetic field would be $\mathrm{B}_{\min } \approx 0.6 \mu \mathrm{G}$, not atypical for WAT lobe emission (see $\S 5.1$ ).

$\mathrm{S}_{\text {Diff }}$ could be caused by ICM or IGM processes, similar to our claim for $\mathrm{N}_{\text {Diff }}$. From Figure 7 we can see that, like $\mathrm{N}_{\text {Diff }}$, there is no cluster X-ray emission detected in either ROSAT 
or XMM. The apparent filament in which $\mathrm{S}_{\text {Diff }}$ is embedded offers an intriguing origin for the diffuse emission. Unlike other diffuse radio sources that have claimed to be part of filamentary large-scale structure (Kim et al. 1989; Giovannini et al. 1990; Kronberg et al. 2007), $\mathrm{S}_{\text {Diff }}$ is not near any massive clusters. At $390 \mathrm{kpc}$ long, $\mathrm{S}_{\text {Diff }}$ is smaller than the $\sim 1.5 \mathrm{Mpc}$ emission in the Coma-Abell 1367 supercluster (Kim et al. 1989; Giovannini et al. 1990) or the $4-5$ Mpc radio regions found by Bagchi et al. (2002) and Kronberg et al. (2007). Bagchi et al. (2002) detected radio emission coincident with a relatively isolated filament of galaxies (in the region of the cluster $\left.\mathrm{ZwCl}_{w} 341.1+0000\right)$, similar to $\mathrm{S}_{\text {Diff }}$. Unlike $\mathrm{S}_{\text {Diff }}$, however, the filament was also detected in X-rays with a $\mathrm{L}_{X}(0.1-2.4 \mathrm{keV}) \approx 10^{44} \mathrm{erg} \mathrm{s}^{-1}$. Though the linear extent of $\mathrm{S}_{\text {Diff }}$ is small compared to the overall size of the filament, it is in the densest region. The radio luminosity of $\mathrm{S}_{\text {Diff }}$ (Table 2 ), assuming it is embedded in the $\mathrm{z} \sim 0.04$ filament, is also several orders of magnitude above the radio vs. X-ray luminosity correlation for known relics (Figure 15). Therefore, if $\mathrm{S}_{\text {Diff }}$ is indeed caused by processes in the ICM or IGM, the lack of X-ray emission poses the same problem as it does for $\mathrm{N}_{\text {Diff }}$. We do not have an active radio galaxy nearby, however, to offer an explanation for $\mathrm{S}_{\text {Diff's }}$ increased radio luminosity.

\subsection{Implications}

A consequence of attributing $\mathrm{N}_{\text {Diff }}$ 's abnormally high radio luminosity to the presence of relic radio plasma is that any relic that forms in the presence of preexisting magnetized plasma will show an increased luminosity. A signature of this scenario might be in the scatter of the currently observed relics, which could be correlated with the availability of relic plasma from current/past AGN activity. However, the Coma relic $1253+275$, which is a relic that is seemingly being fed magnetized plasma from the NAT source NGC 4789 (Enßlin \& Gopal-Krishna 2001), is less radio luminous than other relics with similar X-ray luminosity (Figure 15). This is contrary to our hypothesis that fossil AGN plasma increases the radio luminosity of relic sources. There are, however, many factors that contribute to the presence and strength of synchrotron emission at a structure formation shock. Miniati et al. (2001) found scatter of the same order as Figure 15 for the $1.4 \mathrm{GHz}$ emission of primary electrons in their simulations. This is after integrating the radio luminosity over a spherical volume with a radius of $1.3 h^{-1} \mathrm{Mpc}$, and without including relic plasma from past AGN activity. Since Miniati et al. (2001) used a fixed radius for all the clusters, the radio luminosities they found depend on the details of the current dynamical state and the location of shocks within (or just outside) each cluster. In practice, radio relics represent only a single (perhaps partially) illuminated shock front associated with a cluster. It is thus no surprise that there is such a large scatter in the observational correlation.

What then makes these two sources, $\mathrm{N}_{\text {Diff }}$ and $\mathrm{S}_{\text {Diff }}$, special? Why have radio relics only been found around rich X-ray clusters up until now? Part of the observational problem is most certainly selection and bias effects. Thus far most searches for relics have focused on rich X-ray emitting clusters, an exception being Rudnick, Delain \& Lemmerman (2006), from which 0809+39 was found. 
Even Delain \& Rudnick (2006) found only a handful of new candidates, but this could be the tip of a larger distribution that is currently below the NVSS and WENSS surface-brightness limits. On the theoretical side, most simulations that include synthetic radio observations, e.g., Miniati et al. (2001) and Pfrommer, Enßlin \& Springel (2008), do not include pre-existing relativistic plasma from past/current AGN. Hoeft et al. (2004) simulated the merger of two $\mathrm{M} \approx 1.6 \times 10^{13} M_{\odot}$ clusters of galaxies in order to track the revival of relic radio plasma. They found that efficient re-acceleration only occurred in regions where the ratio of magnetic pressure to gas pressure is $\mathrm{P}_{B} / \mathrm{P}_{\text {gas }}<0.01$, which explains why this process is inefficient in the inner regions of clusters where $\mathrm{P}_{B} \approx \mathrm{P}_{\text {gas }}$. One property that distinguishes $\mathrm{N}_{\text {Diff }}$ and $\mathrm{S}_{\text {Diff }}$ from other known relics is that they reside in poor environments. Perhaps poor clusters and groups do not confine relativistic plasma as well, so that radio lobe plasma from AGN can be driven farther (and faster) into the low density regions than they would in rich clusters (we are speaking here about driven jets, not buoyant forces). For adiabatic compression to be most effective, one wants "younger" electrons, higher compression factors (steeper shocks in cooler gas), and lower magnetic fields (i.e., less radiative losses; Enßlin \& Gopal-Krishna 2001). The first two of these conditions can be effectively achieved in the above scenario. Simulations focusing on the evolution of relic AGN plasma in low-density environments are needed in order to fully assess the plausibility of this idea.

\section{Summary}

We have presented detailed radio observations of the diffuse source $0809+39$ in an attempt to discover the origin of the synchrotron emission. To summarize the key messages:

- Evidence points toward $\mathrm{N}_{\text {Diff }}$ being a radio relic, i.e. shock excited synchrotron emission, related to a poor $\mathrm{z} \sim 0.2$ group of galaxies.

- $\mathrm{S}_{\text {Diff }}$ 's origin is ambiguous, though its coincidence with a filament of galaxies at $\mathrm{z} \sim 0.04$ makes it possible that it could be synchrotron emission from filamentary large-scale structure or relic emission from an extinct radio galaxy within the filament.

- Both $\mathrm{N}_{\text {Diff }}$ and $\mathrm{S}_{\text {Diff }}$ are more radio luminous than their X-ray properties would suggest, given the apparent $\mathrm{P}_{\nu}$ vs. $\mathrm{L}_{X}$ correlation of known radio relics.

- Total energies in $\mathrm{N}_{\text {Diff }}$ and $\mathrm{S}_{\text {Diff }}$ are comparable to luminous, diffuse radio galaxies, and could be the result of adiabatic compression/reacceleration of past AGN activity.

- When analyzing diffuse radio emission beyond the environments of rich galaxy clusters, determining the true physical origin of these structures is non-trivial, especially at very low surface-brightness levels where emission related to large-scale structure is expected.

The issues presented here highlight the difficulty in finding a unified physical model for radio relics. Detailed observations of the radio spectrum, coupled with deep X-ray observations, are needed for a large sample of radio relics in both poor and rich environments in order to determine such a model. 
We gratefully acknowledge help and advice from G. de Bruyn and M. Brentjens during our RM-Synthesis analysis of the WSRT data. We thank K. Delain for help in setting up the VLA and WSRT observations. Partial support for this work at the University of Minnesota comes from the U.S. National Science Foundation grants AST 0307600 and AST 0607674.

The Very Large Array is a facility of the National Science Foundation, operated by NRAO under contract with AUI, Inc. We also acknowledge the use of NASA's SkyView facility 4 located at NASA Goddard Space Flight Center. The Westerbork Synthesis Radio Telescope is operated by the ASTRON (Netherlands Foundation for Research in Astronomy) with support from the Netherlands Foundation for Scientific Research (NWO). The Digitized Sky Surveys were produced at the Space Telescope Science Institute under U.S. Government grant NAG W-2166. Archival observations obtained from XMM-Newton, an ESA science mission with instruments and contributions directly funded by ESA Member States and NASA, and ROSAT archives from HEASARC. Funding for the SDSS and SDSS-II has been provided by the Alfred P. Sloan Foundation, the Participating Institutions, the National Science Foundation, the U.S. Department of Energy, the National Aeronautics and Space Administration, the Japanese Monbukagakusho, the Max Planck Society, and the Higher

Education Funding Council for England. The SDSS Web Site is http://www.sdss.org/. The SDSS is managed by the Astrophysical Research Consortium for the Participating Institutions.

\section{REFERENCES}

Abell, G. O., Corwin, H. G., Jr., \& Olowin, R. P. 1989, ApJS, 70, 1

Adelman-McCarthy, J. K., \& et al. 2007, VizieR Online Data Catalog, 2276, 0

Bagchi, J., Enßlin, T. A., Miniati, F., Stalin, C. S., Singh, M., Raychaudhury, S., \& Humeshkar, N. B. 2002, New Astronomy, 7, 249

Becker, R. H., White, R. L., \& Helfand, D. J. 1995, ApJ, 450, 559

Bell, A. R. 1978, MNRAS, 182, 147

Blandford, R., \& Eichler, D. 1987, Phys. Rep., 154, 1

Blanton, E. L., Gregg, M. D., Helfand, D. J., Becker, R. H., \& White, R. L. 2003, AJ, 125, 1635

Brentjens, M. A., \& de Bruyn, A. G. 2005, A\&A, 441, 1217

Brentjens, M.A. 2007, PhD Thesis

Burn, B. J. 1966, MNRAS, 133, 67

\footnotetext{
4 (http://skyview.gsfc.nasa.gov)
} 
Cioffi, D. F., \& Jones, T. W. 1980, AJ, 85, 368

Clarke, T. E., \& Ensslin, T. A. 2006, AJ, 131, 2900

Condon, J. J., Cotton, W. D., Greisen, E. W., Yin, Q. F., Perley, R. A., Taylor, G. B., \& Broderick, J. J. 1998, AJ, 115, 1693

Csabai, I., et al. 2003, AJ, 125, 580

de Bruyn, A. G., \& Brentjens, M. A. 2005, A\&A, 441, 931

Delain, K. M., \& Rudnick, L. 2006, Astronomische Nachrichten, 327, 561

De Young, D. S. 1984, Phys. Rep., 111, 373

Drury, L. 1983, Space Science Reviews, 36, 57

Enßlin, T. A., \& Gopal-Krishna 2001, A\&A, 366, 26

Feretti, L. 2003, ArXiv Astrophysics e-prints, arXiv:astro-ph/0301576

Feretti, L. 2006, ArXiv Astrophysics e-prints, arXiv:astro-ph/0612185

Gal, R. R., de Carvalho, R. R., Lopes, P. A. A., Djorgovski, S. G., Brunner, R. J., Mahabal, A., \& Odewahn, S. C. 2003, AJ, 125, 2064

Giovannini, G., Kim, K. T., Kronberg, P. P., \& Venturi, T. 1990, Galactic and Intergalactic Magnetic Fields, 140, 492

Giovannini, G., Feretti, L., \& Stanghellini, C. 1991, A\&A, 252, 528

Giovannini, G., \& Feretti, L. 2000, New Astronomy, 5, 335

Giovannini, G., \& Feretti, L. 2004, Journal of Korean Astronomical Society, 37, 323

Giovannini, G., Tordi, M., \& Feretti, L. 1999, New Astronomy, 4, 141

Gomez, P. L., Pinkney, J., Burns, J. O., Wang, Q., Owen, F. N., \& Voges, W. 1997, ApJ, 474, 580

Govoni, F., Feretti, L., Giovannini, G., Böhringer, H., Reiprich, T. H., \& Murgia, M. 2001, A\&A, 376,803

Govoni, F., Murgia, M., Feretti, L., Giovannini, G., Dallacasa, D., \& Taylor, G. B. 2005, A\&A, 430, L5

Hartley, W. G., Gazzola, L., Pearce, F. R., Kay, S. T., \& Thomas, P. A. 2008, MNRAS, 386, 2015

Hoeft, M., Brüggen, M., \& Yepes, G. 2004, MNRAS, 347, 389 
Kempner, J. C., Blanton, E. L., Clarke, T. E., Enßlin, T. A., Johnston-Hollitt, M., \& Rudnick, L. 2004, The Riddle of Cooling Flows in Galaxies and Clusters of galaxies, 335

Kempner, J. C., \& Sarazin, C. L. 2001, ApJ, 548, 639

Kim, K.-T., Kronberg, P. P., Giovannini, G., \& Venturi, T. 1989, Nature, 341, 720

Kronberg, P. P., Burbidge, E. M., Smith, H. E., \& Strom, R. G. 1977, ApJ, 218, 8

Kronberg, P. P., Kothes, R., Salter, C. J., \& Perillat, P. 2007, ApJ, 659, 267

Ledlow, M. J., Voges, W., Owen, F. N., \& Burns, J. O. 2003, AJ, 126, 2740

Loken, C., Roettiger, K., Burns, J. O., \& Norman, M. 1995, ApJ, 445, 80

Machalski, J., Jamrozy, M., \& Zola, S. 2001, A\&A, 371, 445

Markevitch, M., Govoni, F., Brunetti, G., \& Jerius, D. 2005, ApJ, 627, 733

Merchán, M. E., \& Zandivarez, A. 2005, ApJ, 630, 759

Micono, M., Zurlo, N., Massaglia, S., Ferrari, A., \& Melrose, D. B. 1999, A\&A, 349, 323

Miller, C. J., et al. 2005, AJ, 130, 968

Miniati, F., Jones, T. W., Kang, H., \& Ryu, D. 2001, ApJ 562, 233

Owen, F. N., \& Rudnick, L., 1976, ApJ 205, 1

Parma, P., Murgia, M., de Ruiter, H. R., Fanti, R., Mack, K.-H., \& Govoni, F. 2007, A\&A, 470, 875

Pinkney, J. 1993, BAAS, 25, 1437

Pinkney, J., Burns, J. O., Ledlow, M. J., Gómez, P. L., \& Hill, J. M. 2000, AJ, 120, 2269

Pfrommer, C., Enßlin, T. A., \& Springel, V. 2008, MNRAS, 385, 1211

Popesso, P., Böhringer, H., Brinkmann, J., Voges, W., \& York, D. G. 2004, A\&A, 423, 449

Reich, W. 2006, ArXiv Astrophysics e-prints, arXiv:astro-ph/0603465

Roettiger, K., Burns, J. O., \& Loken, C. 1996, ApJ, 473, 651

Rudnick, L., Delain, K. \& Lemmerman, J., 2006, AN 327, 549

Rudnick, L., \& Lemmerman, J., 2008, submitted to ApJ

Ryu, D., Kang, H., Hallman, E., \& Jones, T. W. 2003, ApJ, 593, 599 
Sadler, E. M., et al. 2007, MNRAS, 381, 211

Sarazin, C. L. 1999, ApJ, 520, 529

Schmidt, S. J., Connolly, A. J., \& Hopkins, A. M. 2006, ApJ, 649, 63

Slee, O. B., Roy, A. L., Murgia, M., Andernach, H., \& Ehle, M. 2001, AJ, 122, 1172

Stoughton, C., et al. 2002, AJ, 123, 485

York, D. G., et al. 2000, AJ, 120, 1579 


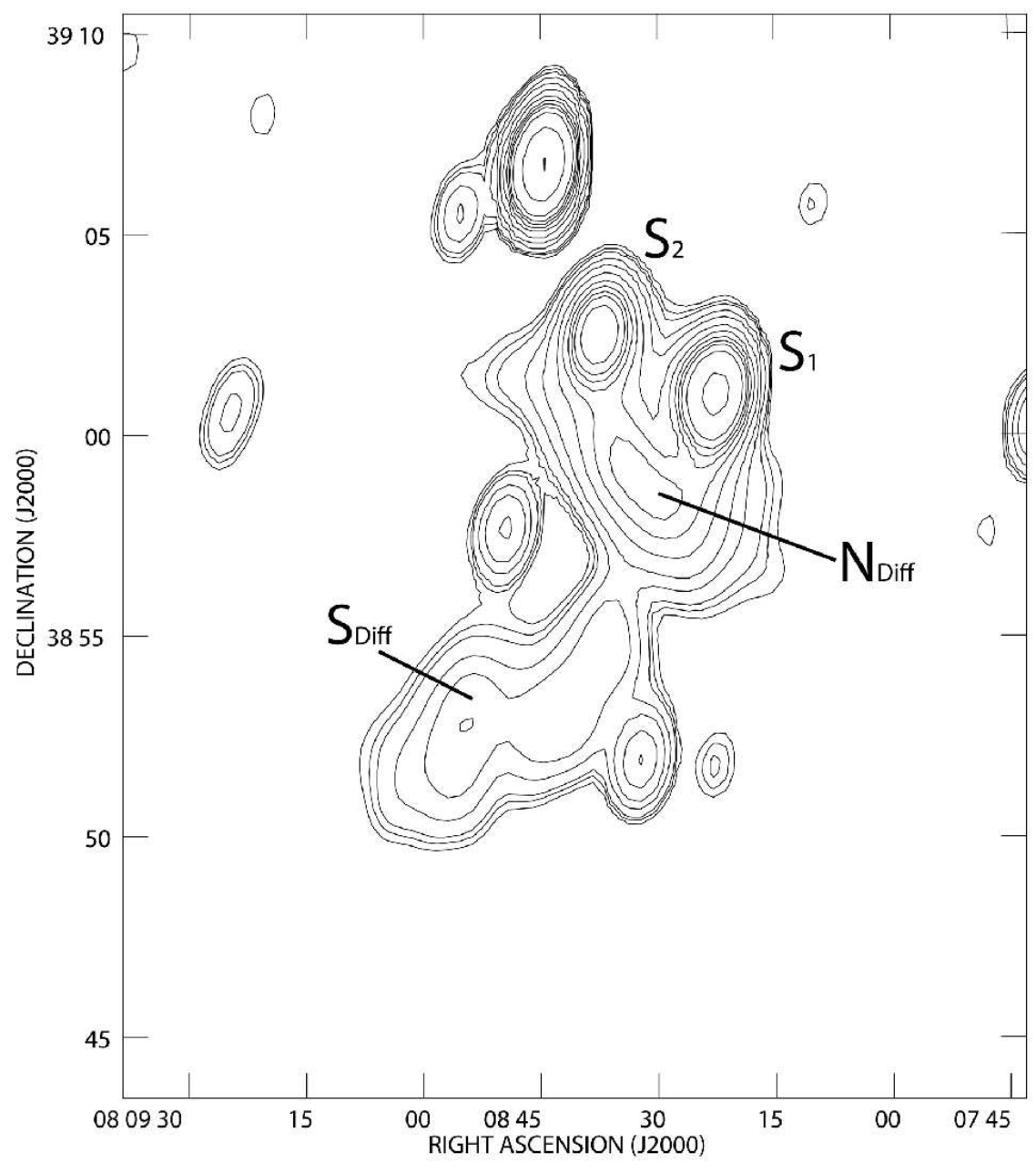

Fig. 1. - WSRT $351 \mathrm{MHz}$ total intensity image with contour levels $8.254 \times 10^{-3}(-0.4,0.4,0.5,0.8$, $1.2,2,3,4,6,8,16,32,64) \mathrm{Jy} /\left(108^{\prime \prime} \times 60^{\prime \prime}\right.$ beam $)$. S1 and $\mathrm{S} 2$ are "compact" sources at $351 \mathrm{MHz}$ that show sub-structure at higher resolution (see Figure 21). 


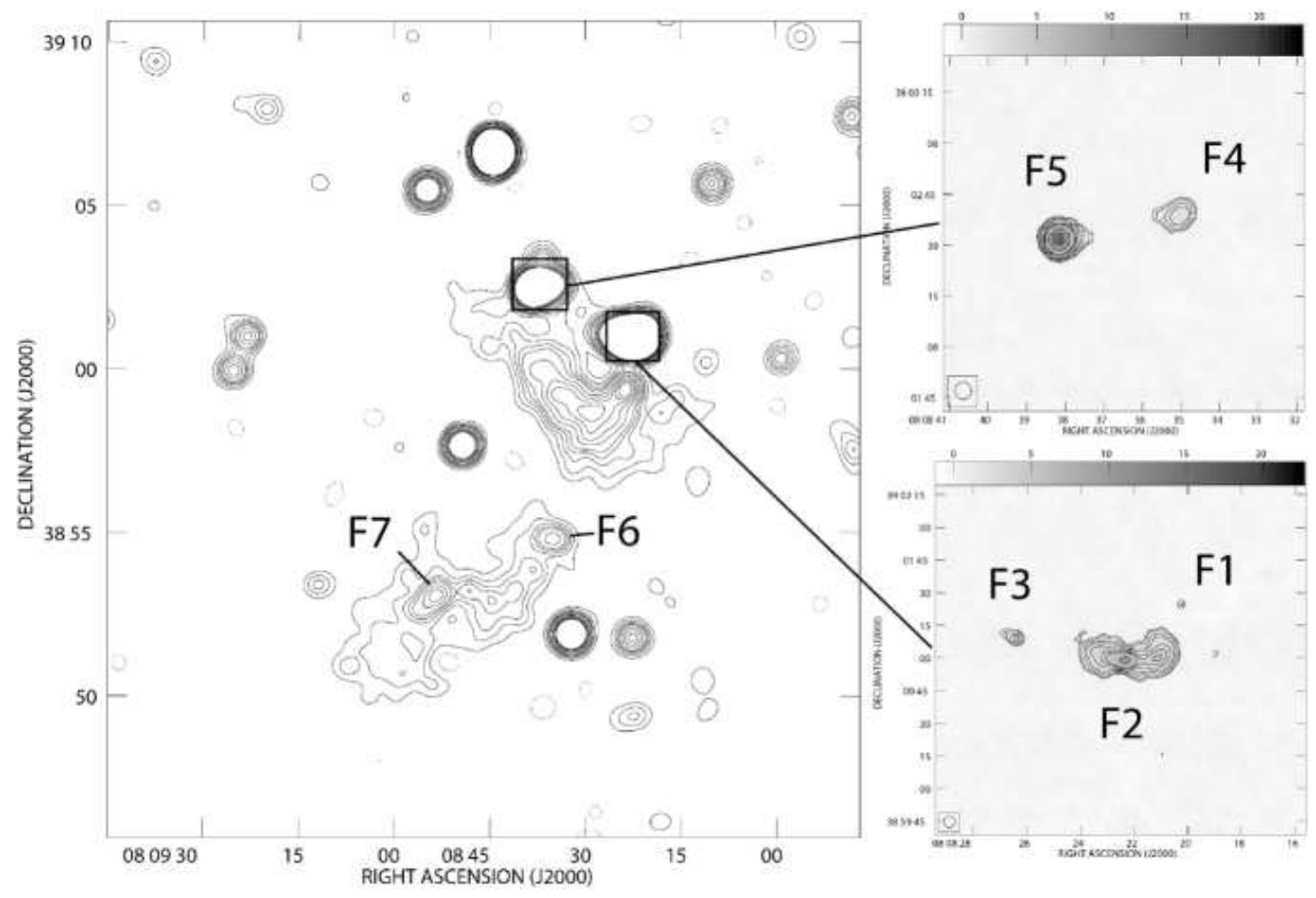

Fig. 2.- VLA I image (left) with contours $2.0 \times 10^{-4}(-1,1,2,3,4,5,7,9,11,13,15) \mathrm{Jy} /\left(40^{\prime \prime} \times 40^{\prime \prime}\right.$ beam). At right are close-ups views of S1 and S2 from the FIRST survey. Contours $4.0 \times 10^{-4}(1.5$, $2,3,4,6,8,10,20,50,100) \mathrm{Jy} /\left(5^{\prime \prime} \times 5^{\prime \prime}\right.$ beam $)$. 


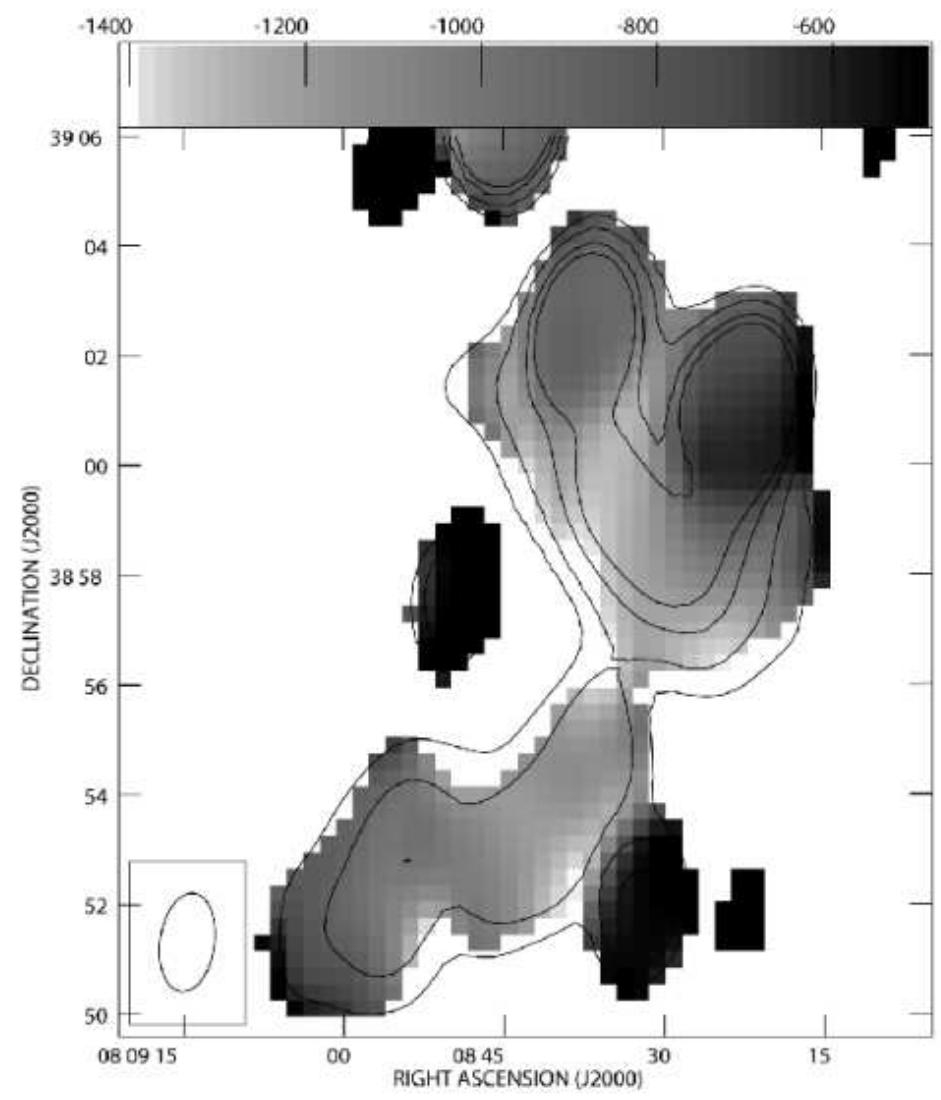

Fig. 3.- Spectral index map from $351 \mathrm{MHz}$ to $1.4 \mathrm{GHz}$, with WSRT contours $8.25 \times 10^{-3}(-0.5,0.5$, $0.8,1,2,3,6,12,24,48) \mathrm{Jy} /\left(108^{\prime \prime} \times 60^{\prime \prime}\right.$ beam $)$. 

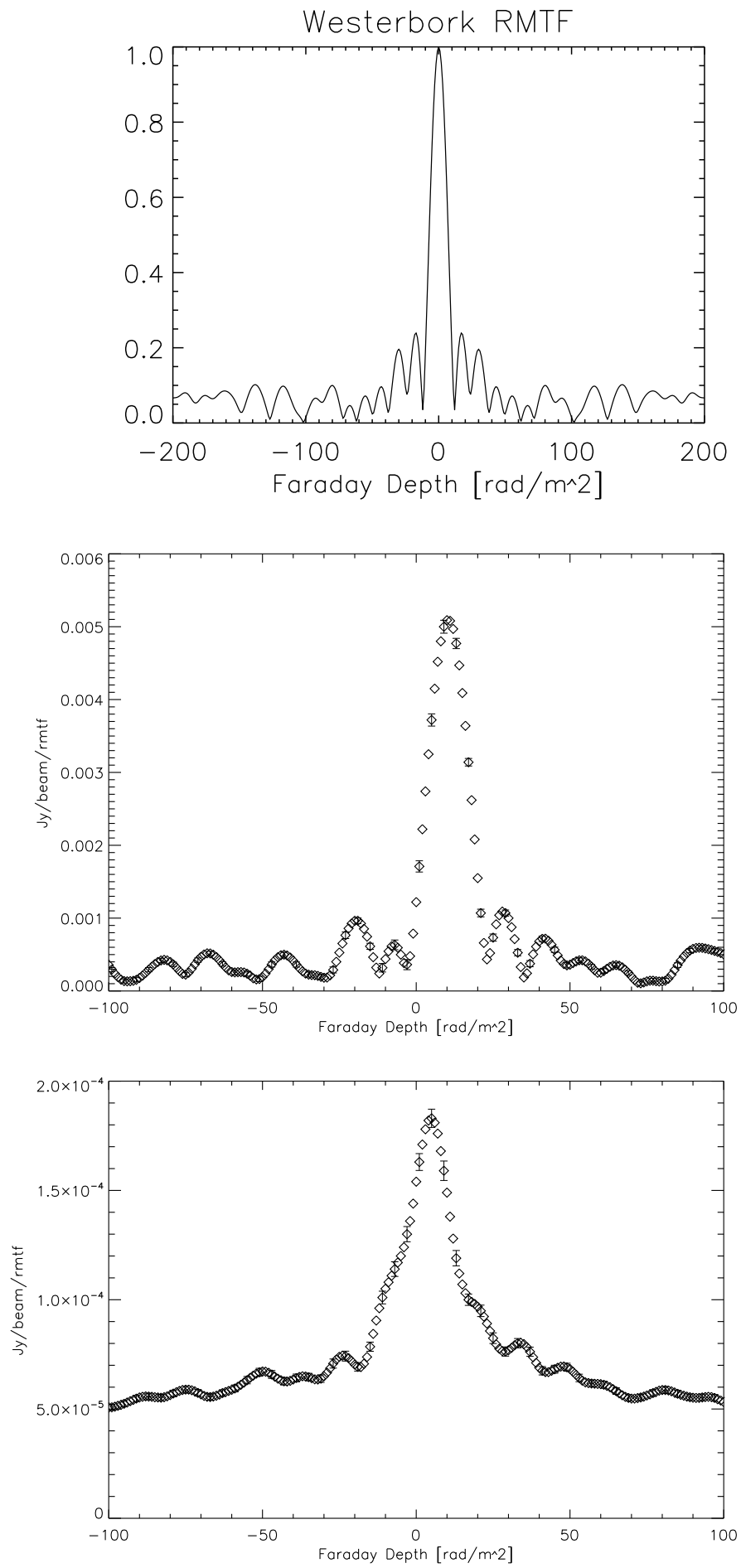

Fig. 4.- Top: Our RMTF; Center: Faraday spectrum for $\mathrm{N}_{\text {Diff }}$; Bottom: Faraday spectrum for a large area of the Galactic emission. Characteristic error bars are shown. 


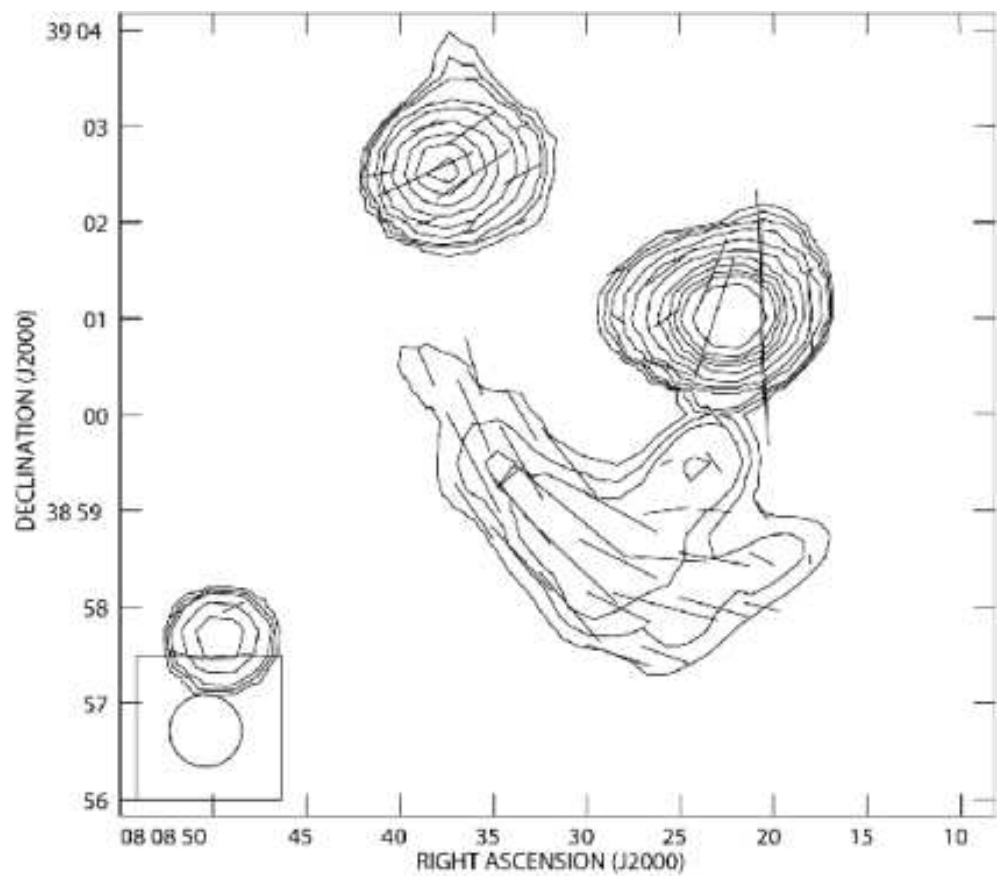

Fig. 5.- B field orientation obtained from derotating the NVSS to correct for a rotation measure of $+12 \mathrm{rad} \mathrm{m}^{-2}$. Contours are NVSS I at $4.9 \times 10^{-3}(-0.3,0.3,0.4,0.5,0.8,1.2,2,3,4,5,6,8,16$, $32,64) \mathrm{Jy} /\left(45^{\prime \prime} \times 45^{\prime \prime}\right.$ beam $)$, and the magnetic field lines are $1^{\prime \prime}=2.22 \times 10^{-5} \mathrm{Jy} /\left(45^{\prime \prime} \times 45^{\prime \prime}\right.$ beam $)$. 


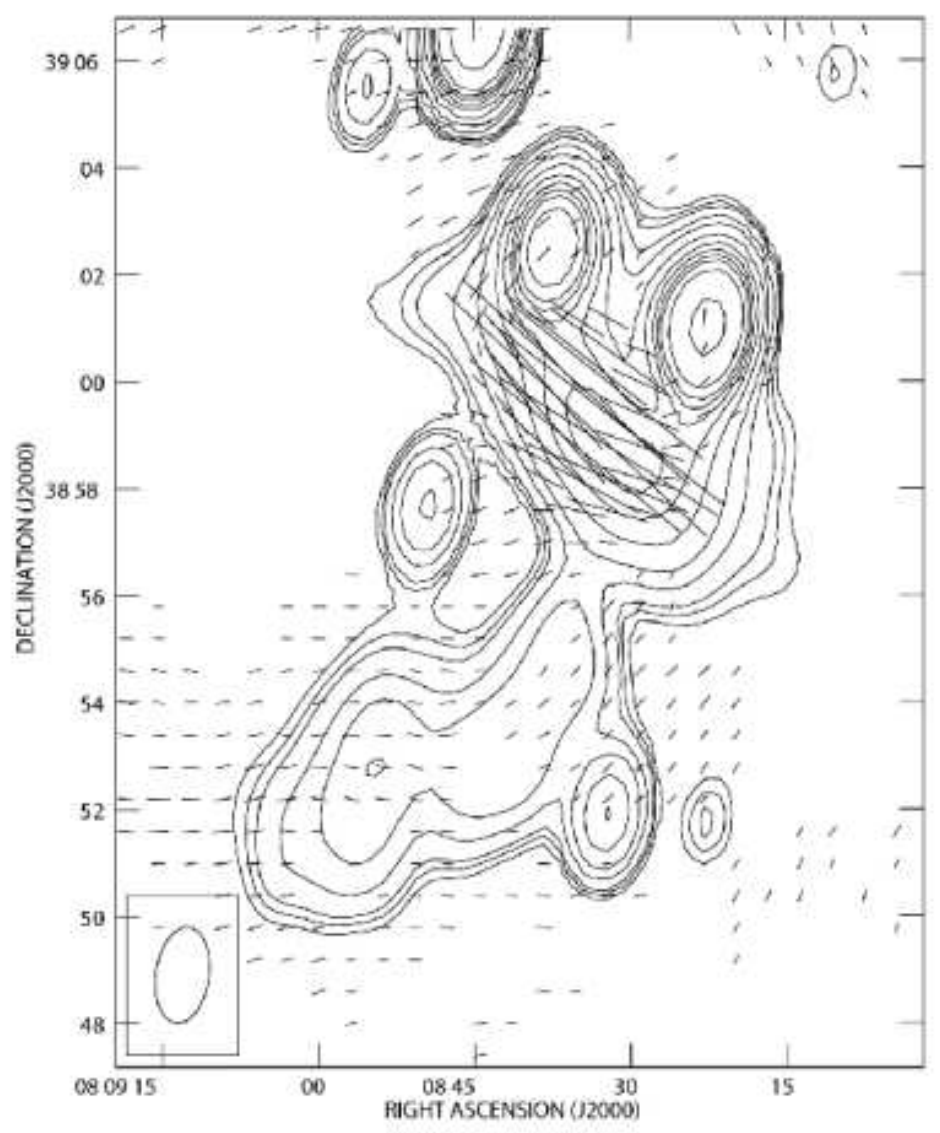

Fig. 6.- WSRT $351 \mathrm{MHz}$ contour image of 0809+39 I (same contours and resolution as Figure 1) with corrected magnetic field vectors for the RM-Cube at $\phi=+12 \mathrm{rad} \mathrm{m}^{-2}$. $1^{\prime \prime}=$ $1.85 \times 10^{-5} \mathrm{Jy} /\left(108^{\prime \prime} \times 60^{\prime \prime}\right.$ beam $)$. The apparent "noise" in the polarized emission is actually real emission from our own galaxy. 


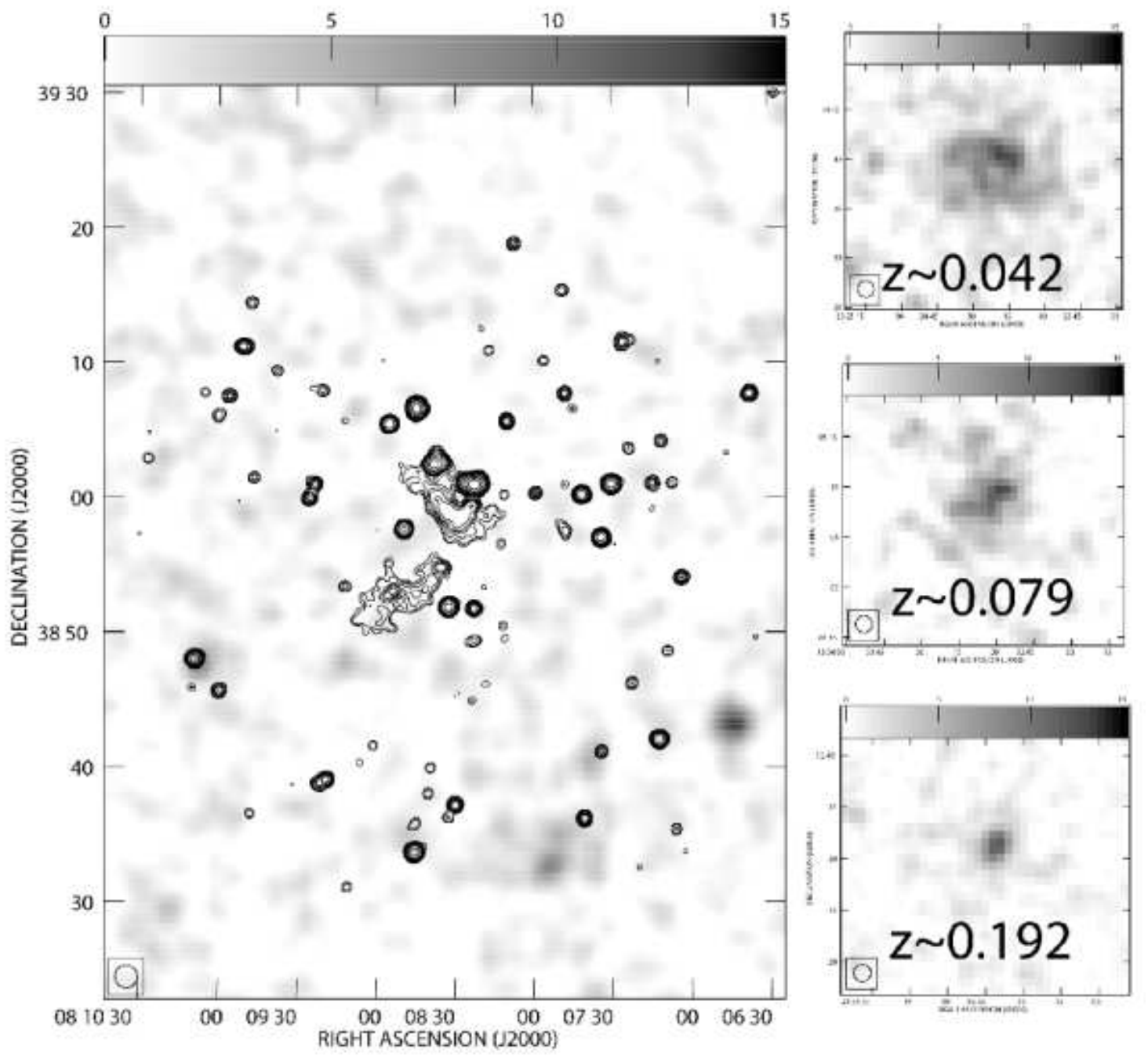

Fig. 7.- ROSAT broad (0.1-2.4 keV) continuum grayscale (convolved to 100") with VLA L-band contours. To the right are ROSAT images of three X-ray selected clusters at the indicated redshifts, at the same grayscale and resolution as the 0809+39 ROSAT image. 

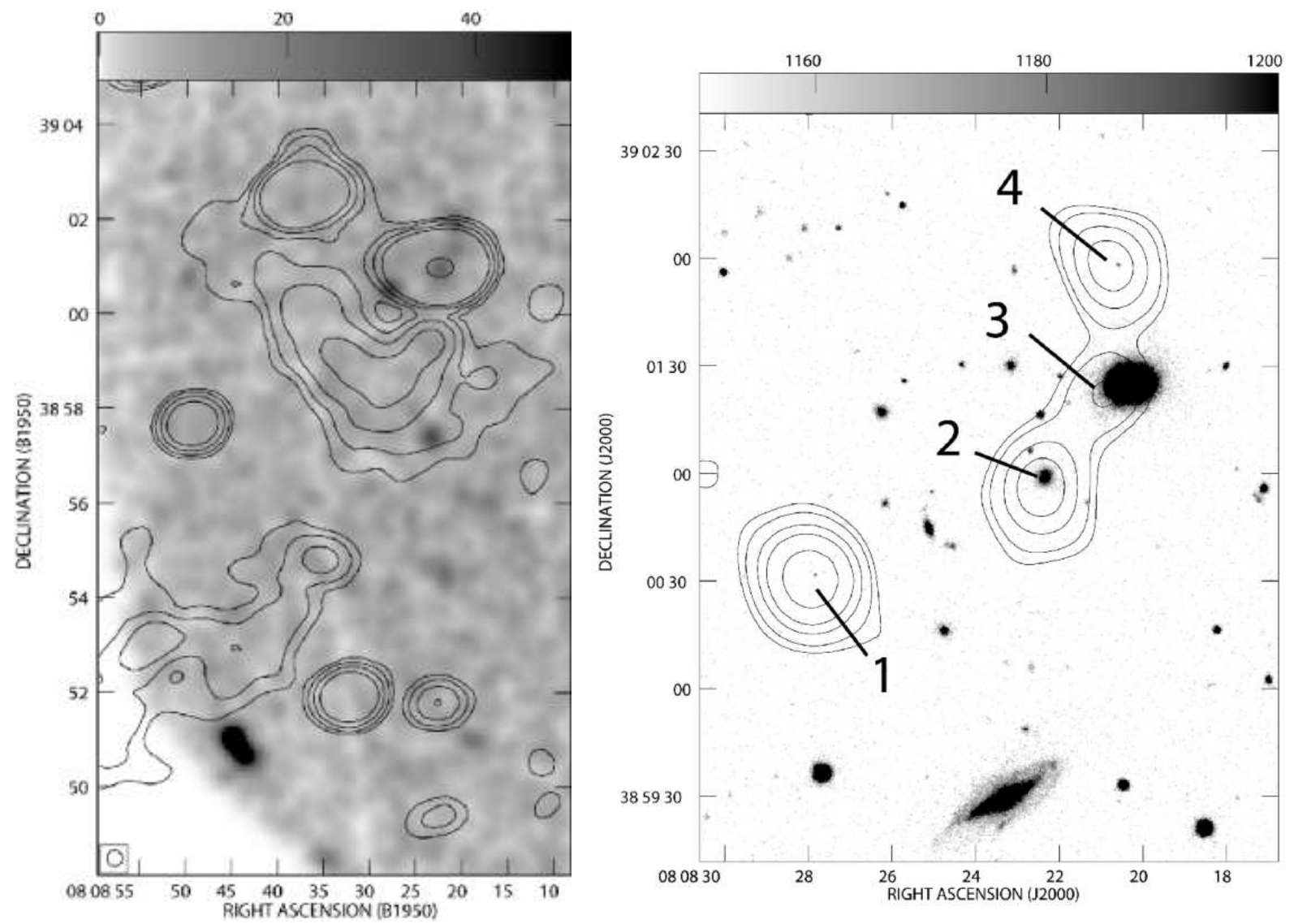

Fig. 8. - Left: XMM Epic observation in counts (convolved to 20") with VLA radio contours $6.5 \times 10^{-3}(0.05,0.1,0.2,0.4,8) \mathrm{Jy} /\left(49^{\prime \prime} \times 42^{\prime \prime}\right.$ beam $)$. Note that a faint X-ray source is coincident with F2 (the WAT seen in Figure 2); Right: SDSS R image with XMM EPIC contours. Grayscale is in units of counts, and the contours are $4.08 \times(3.5,4,5,6,8)$ counts. X-ray source 2 is the WAT (F2) and the galaxy near source 3 is coincident with compact radio source F1. 


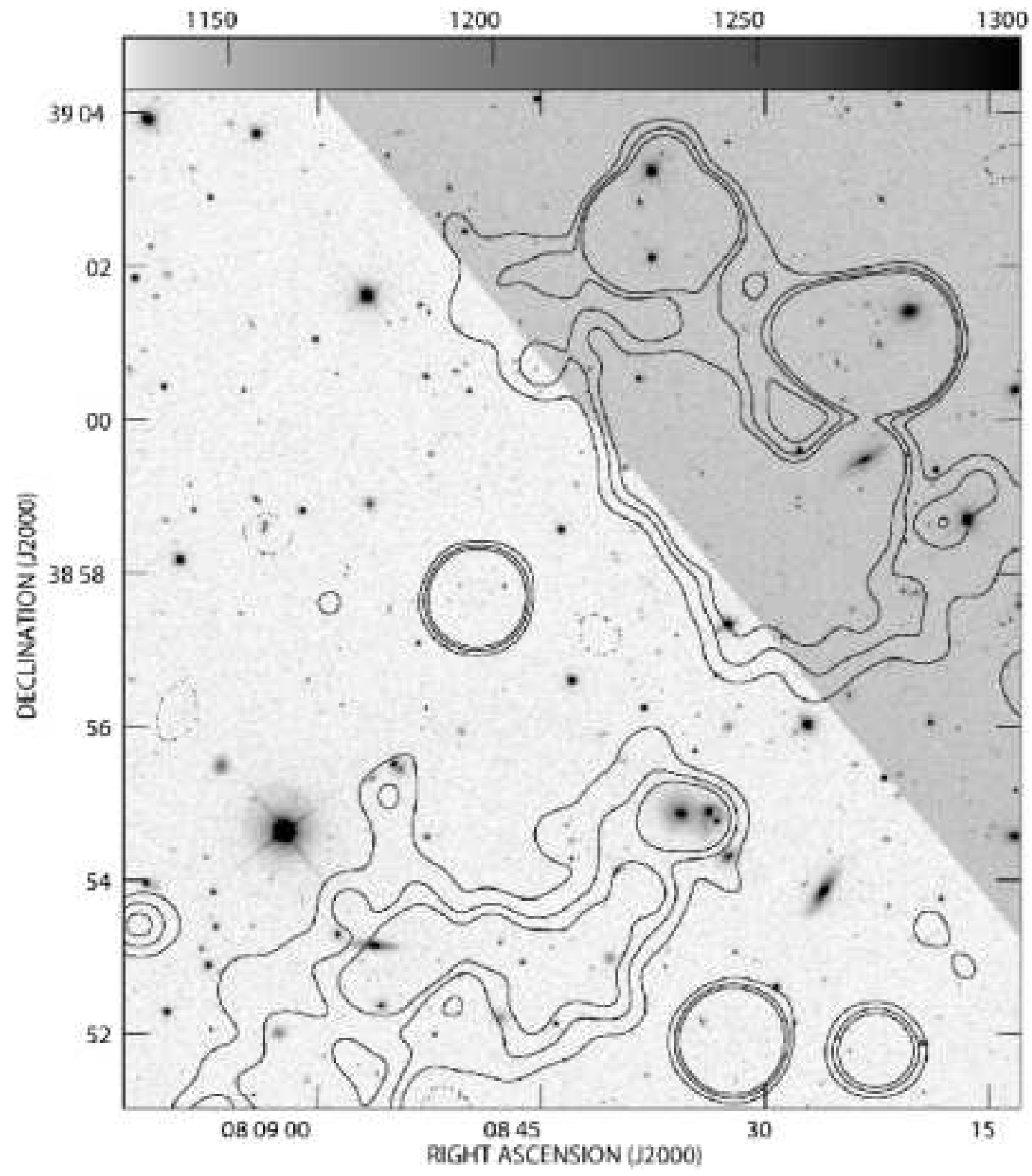

Fig. 9. - SDSS R mosaic grayscale (in counts) with VLA $1.4 \mathrm{GHz}$ contours at $2.0 \times 10^{-4}(-1,1,2$, 3) $\mathrm{Jy} /\left(40^{\prime \prime} \times 40^{\prime \prime}\right.$ beam $)$. 


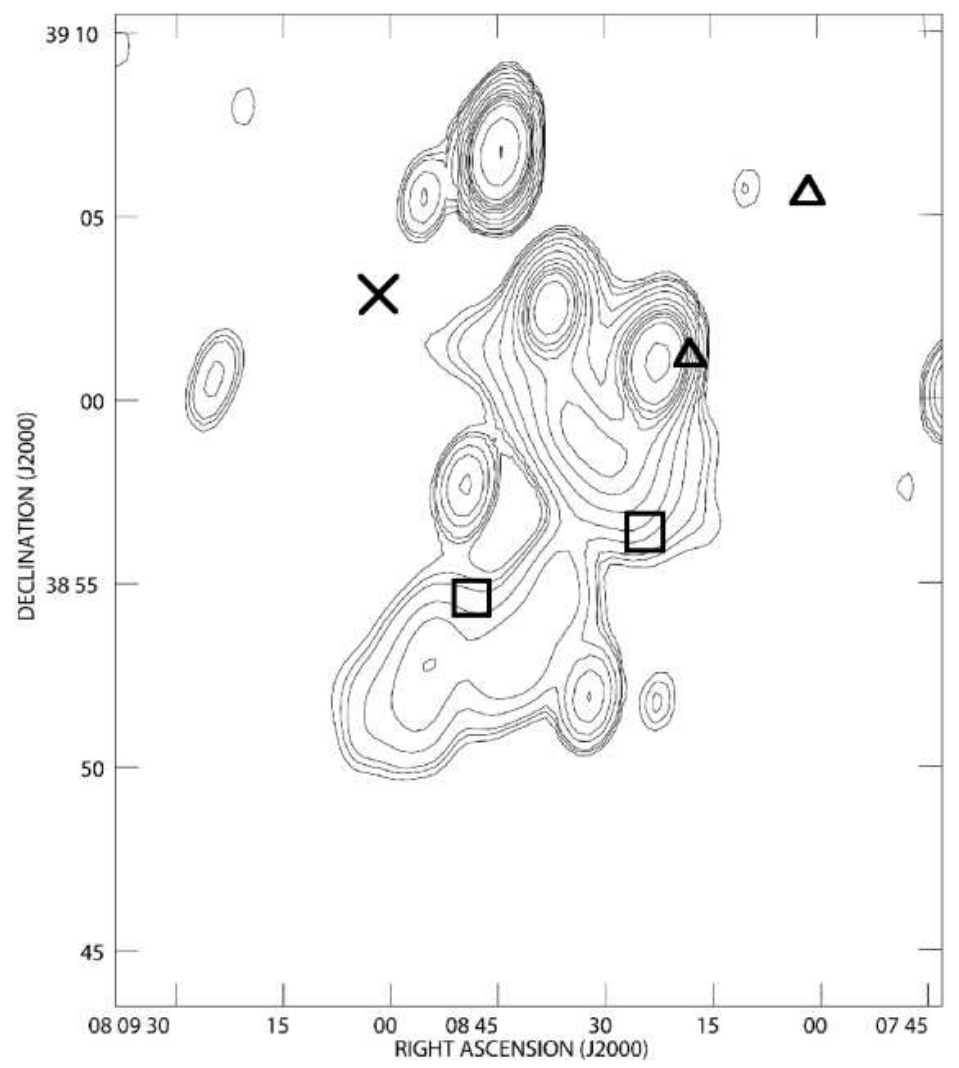

Fig. 10.- Plot of known groups/clusters in the $0809+39$ region. Boxes are $\mathrm{z} \approx 0.04$ systems, triangles are $\mathrm{z} \approx 0.07$ systems, and the cross is a $\mathrm{z} \approx 0.11$ cluster. 

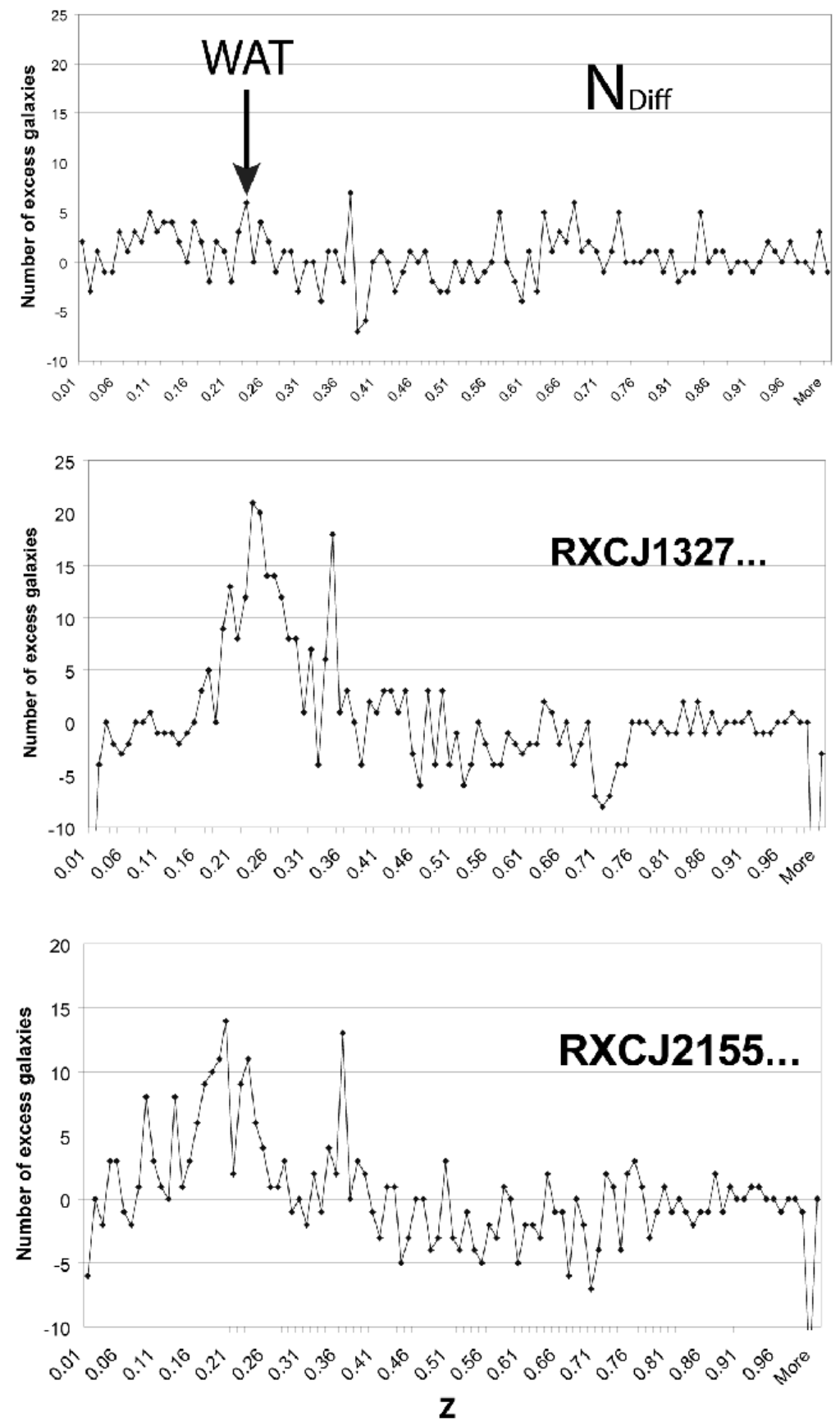

Fig. 11. - Histograms of galaxy photometric redshifts from SDSS for $6^{\prime}$ around the WAT source F2 (Top, see Figure 2) and the X-ray selected clusters RXCJ1327.0+0211 (Center) and RXCJ2155.6+1231 (Bottom) (Popesso et al. 2004). Note that all the histograms have a narrow peak at $\mathrm{z} \sim 0.35$. This is an artifact of the template fitting method for calculating the photometric redshifts (Csabai et al. 2003; see Figure 16 in that paper). 

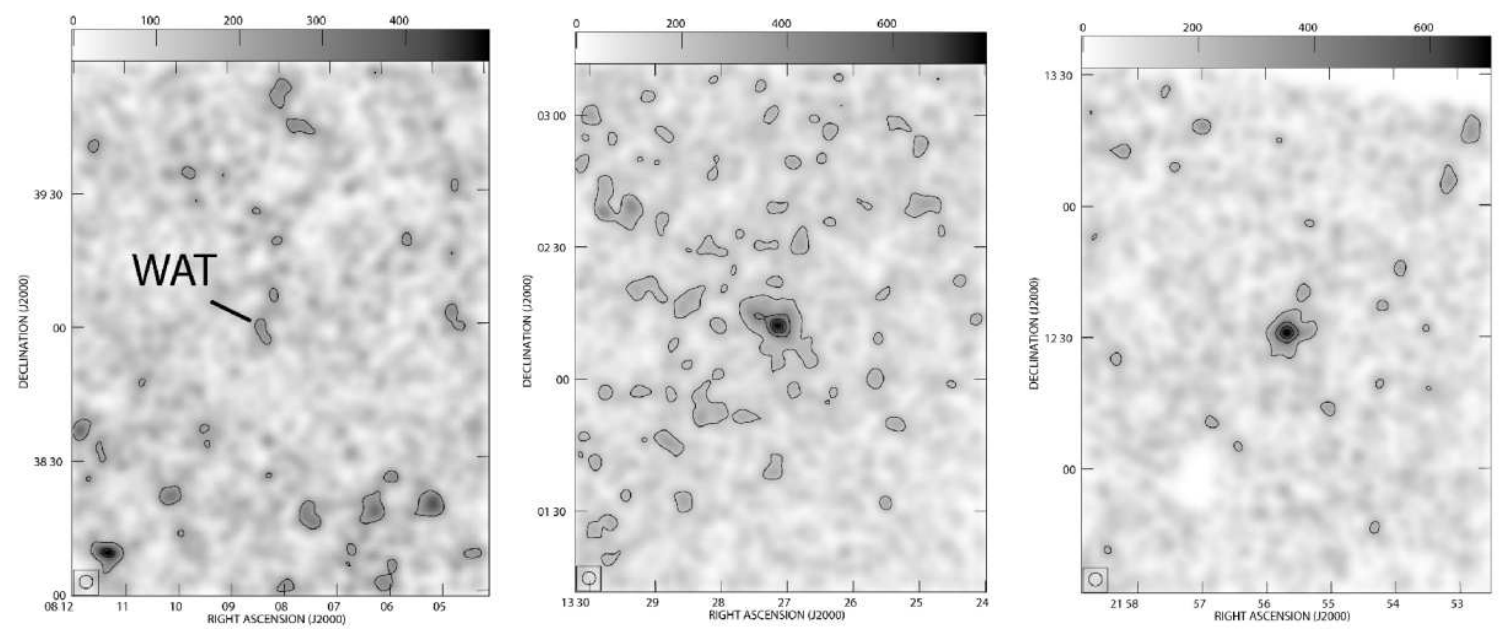

Fig. 12.- Distribution of galaxies from the SDSS photometric database with redshifts between $0.15<\mathrm{z}<0.25$, smoothed to $6^{\prime}$. Left: $0809+39$; Using the same redshift limits we show two X-ray selected clusters for comparison: Middle: RXCJ1327.0+0211; Right: RXCJ2155.6+1231 

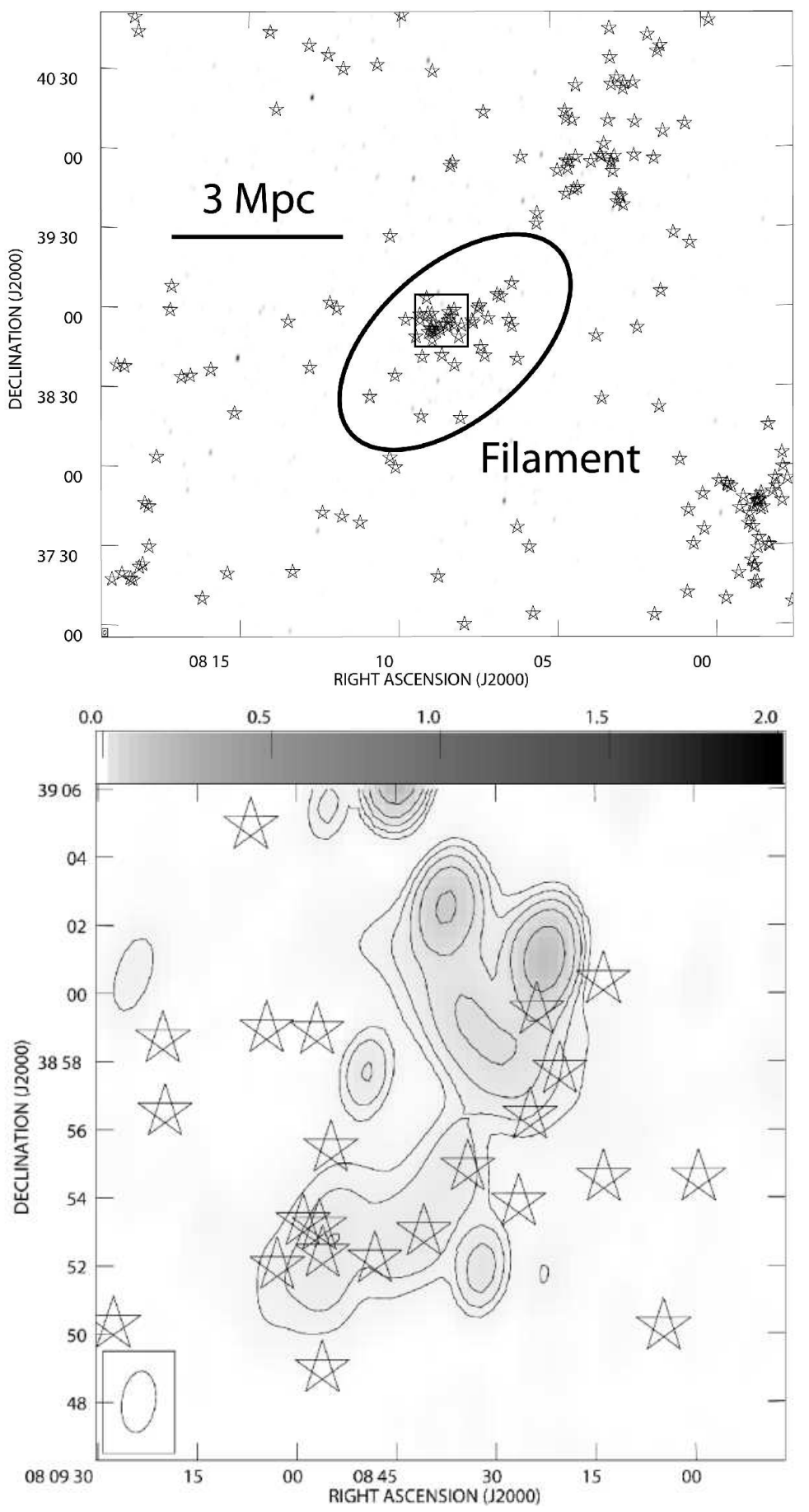

Fig. 13. - Top: Distribution of $0.0366<\mathrm{z}<0.0448$ galaxies with spectroscopic red-shifts in SDSS plotted as stars. Bottom: close-up view of the boxed region in the top panel. $\mathrm{S}_{\text {Diff }}$ is embedded in the filament of galaxies. 


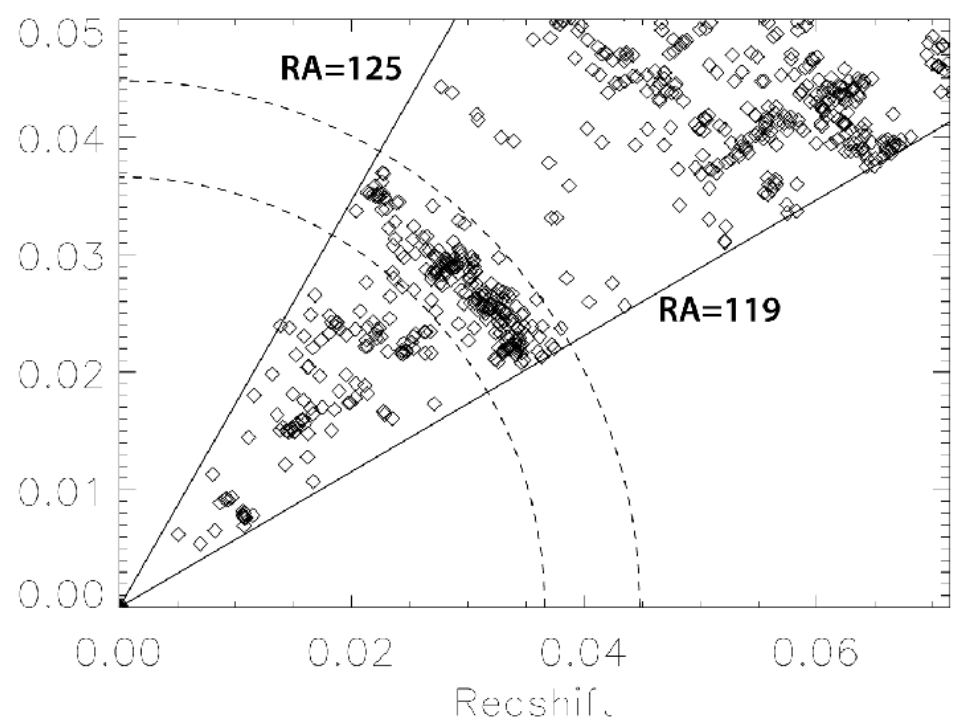

Fig. 14.- Distribution of redshift vs. RA for galaxies with spectroscopic redshifts in SDSS in a roughly $4 \times 4$ degree field around $0809+39$. The dashed lines enclose the range in $\mathrm{z}$ used to create Figure $13(0.0366<\mathrm{z}<0.0448)$, and show the clustering of the filament galaxies. There are other significant structures at higher redshifts, but the filament at $\mathrm{z}=0.04$ was deemed significant based on its spatial correlation with $\mathrm{S}_{\text {Diff }}$. 


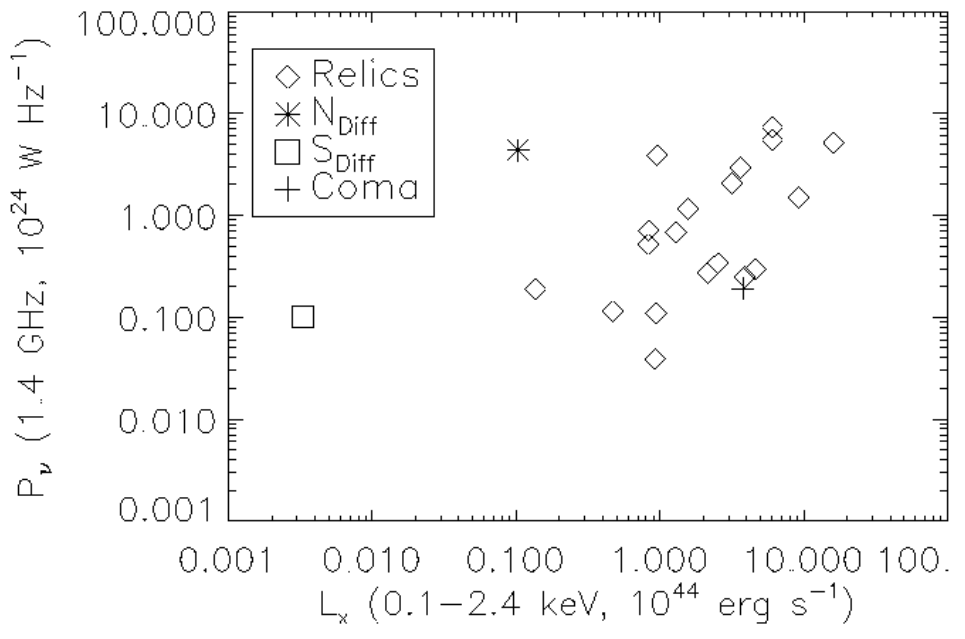

Fig. 15. - Plot of relic radio luminosity/Hz at $1.4 \mathrm{GHz}$ vs. the $0.1-2.4 \mathrm{keV}$ X-ray luminosity of the associated cluster. Compiled are 22 radio relics from Giovannini et al. (1991); Giovannini, Tordi, \& Feretti (1999); Kempner \& Sarazin (2001); Govoni et al. (2001); Slee et al. (2001); Govoni et al. (2005). They represent a complete list of radio relics that have reasonably reliable $1.4 \mathrm{GHz}$ Flux density measurements. 


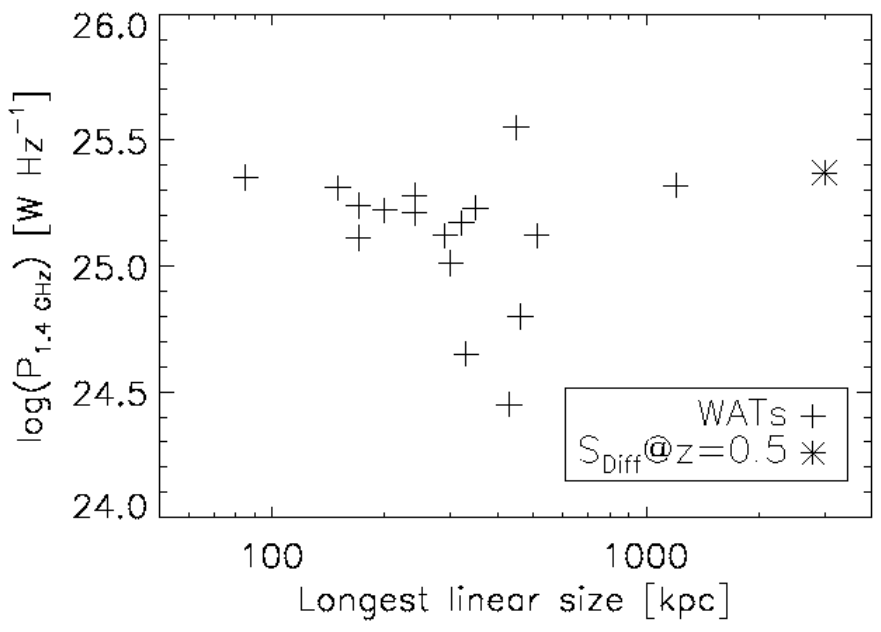

Fig. 16.- $1.4 \mathrm{GHz}$ power vs. linear extent in kpc for a sample of WAT sources (Pinkney et al. 2000). $\mathrm{S}_{\text {Diff }}$, if at redshift of $\mathrm{z}>0.5$, is an extremely large radio galaxy. 
Table 1. Properties of the Diffuse Components of $0809+39$

\begin{tabular}{ccccccc}
\hline \hline Source & $\begin{array}{c}\text { P-band flux } \\
(\mathrm{mJy})\end{array}$ & $\begin{array}{c}\text { L-band flux } \\
(\mathrm{mJy})\end{array}$ & $\begin{array}{c}\text { FWHM Size } \\
\text { " }\end{array}$ & $\begin{array}{c}\text { Frac. Pol. 351 MHz } \\
\%\end{array}$ & Mean $\alpha$ & $\begin{array}{c}\phi \\
\text { rad } \mathrm{m}^{-2}\end{array}$ \\
\hline $\mathrm{N}_{\text {Diff }}$ & $178 \pm 0.7$ & $37.8 \pm 0.7$ & 200 & 19 & -1.12 & +12 \\
$\mathrm{~S}_{\text {Diff }}$ & $136 \pm 1.1$ & $24.8 \pm 1.0$ & 490 & $<9(3 \sigma)$ & -1.23 & $\sim+6^{\mathrm{b}}$ \\
\hline
\end{tabular}

${ }^{\text {a }}$ Errors taken from final maps $\sigma_{r m s}$ and does not include the uncertainty in the total intensity calibration

${ }^{\mathrm{b}}$ Typical local galactic value

Table 2. Distance Dependent Properties of 0809+39

\begin{tabular}{ccccccc}
\hline \hline Region & $\mathrm{z}$ & $\begin{array}{c}P_{0.32} \\
10^{23} \mathrm{~W} \mathrm{~Hz}^{-1}\end{array}$ & $\begin{array}{c}P_{1.4} \\
10^{23} \mathrm{~W} \mathrm{~Hz}^{-1}\end{array}$ & $\begin{array}{c}L_{X}(0.1-2.4 \mathrm{keV}) \\
\log \left(\mathrm{erg} \mathrm{s}^{-1}\right)\end{array}$ & $\begin{array}{c}\text { Physical Size } \\
\mathrm{Mpc}\end{array}$ & $\begin{array}{c}B_{\min } \\
\mu \mathrm{G}\end{array}$ \\
\hline $\mathrm{N}_{\text {Diff }}$ & 0.20 & 205 & 43.4 & $<43.0(3 \sigma)$ & 0.66 & 0.64 \\
$\mathrm{~S}_{\text {Diff }}$ & 0.04 & 5.07 & 0.99 & $<41.5(3 \sigma)$ & 0.39 & 0.57 \\
\hline
\end{tabular}

